.

\title{
CFD Analysis for the Performance of Micro-vortex Generator on Aerofoil and Vertical Axis Turbine
}

\author{
Yan Yan ${ }^{1}$, Eldad Avital ${ }^{2}$, John Williams ${ }^{3}$ \\ School of Engineering and Materials Science \\ Queen Mary University of London \\ 327 Mile End Road London E1 4NS UK \\ yan.yan@qmul.ac.uk ${ }^{1}$, e.avital@qmul.ac.uk ${ }^{2}$,j.j.r.williams@qmul.ac.uk ${ }^{3}$ \\ Jiahuan Cui \\ School of Aeronautics and Astronautics, and ZJU-UIUC Institute \\ Zhejiang University \\ Hangzhou 310007, China \\ Jiahuancui@intl.zju.edu.cn
}

\begin{abstract}
A numerical study was carried out to investigate the effects of Micro-Vortex Generators (MVGs) on the aerodynamic performance of the NACA 0018 aerofoil and an H-type Darrieus wind turbine. MVGs can delay stall, which may occur for a sustained duration during turbine operation. The flow fields around a single aerofoil and the Vertical Axis Wind Turbine (VAWT) rotor are investigated. The purpose of the present work is to determine the best configuration of MVGs. In total, eight different configurations are studied. The results show that MVGs have significantly enhanced the lift of the aerofoil near the stall and improve the stall margin. The improved aerofoil design with MVGs installed at $20 \%$ chord length and $16^{\circ}$ to the inlet flow with a rectangle shape has the maximum lift and stall angle. In addition, adding MVGs of the same configuration can significantly improve the power coefficient of the VAWT at high tip speed ratio, where it typically gives low power production. The flow separation is suppressed in the azimuth angle ranging from $120^{\circ}$ to $135^{\circ}$, where the power output increase is observed showing potential impact for VAWT design.
\end{abstract}

Keywords: Vertical axis wind turbine; Micro vortex generator; Aerofoil; Flow control; Lift; Stall

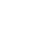

$\mathrm{AoA}$
$\mathrm{c}$
$\mathrm{CFD}$
$C_{D}$
$C_{f}$
$C_{L}$
$C_{m}$
$C_{p}$
$C_{P}$
$\mathrm{e}$
$\mathrm{h}$
$\mathrm{H}$
$\mathrm{HAWT}$

\section{List of symbols}

angle of attack

aerofoil chord length

computational fluid dynamics

drag coefficient

skin driction coefficient

lift coefficient

moment coefficient

pressure coefficeint

power coefficeint

length of micro vortex generator

height of micro vortex generator

height of turbine blade

horizontal axis wind turbine

*Corresponding Author: Eldad Avital e.avital@qmul.ac.uk 


ILES
LES
MVG
R
$R e_{c}$
s
SVG
TSR
(U)RANS
V
VAWT
VG
$\alpha$
$\beta$
$\omega$
$\delta$
$\theta$
$\lambda$

implicit large eddy simulation

large eddy simulation

micro vortex generator

radius of rotor

Reynolds number based on reference chord c distance to the trailing edge of vortex generator smart vortex generators

tip speed ratio

unsteady Reynolds-averaged Navier-Stokes

wind speed

vetical axis wind turbine

vortex generator

angle of attack

installed angle

rotor rotation speed

thickness of boundary layer

azimuth angle

tip speed ratio

\section{INTRODUCTION}

In recent years, wind energy through utility scale wind turbines account for large part in the total renewable power capacity worldwide [1]. Small wind turbines are widely used in various applications for power generation [2]. Among small wind power configurations, the vertical axis wind turbines (VAWTs) offer some unique advantages that horizontal axis wind turbines (HAWTs) do not have. They eliminate the dependence of power production on the incoming direction of the wind. In addition, they can tolerate a wider range of wind velocity and produce lower noise [3]. They also feature a simpler mechanical structure, which is easy to maintain and integrate with buildings [4]. However, VAWTs offer a relatively low power coefficient compared to traditional HAWTs. Hence, there is a strong interest to incorporate flow control techniques to improve the aerodynamic performance of VAWTs.

Passive vortex generators (VGs) have been widely-used flow control devices for various aerodynamic applications, especially in the wind turbine industry, for many years and were firstly introduced by Taylor [5] [8]. He proposed a simple device installed in a diffuser, which consisted of a row of small plates projecting normal to the surface at an installed angle to the free stream airflow. The main function of the VGs is to transfer momentum from the main stream to the inner boundary layer, in order to suppress flow separation. They were also used for enhancing wing lift, reducing noise generated by airflow separation and reducing afterbody drag of aircraft fuselages [6].

Many researchers have studied the mechanism of VGs on aerofoils using both experimental and Computational Fluid Dynamics (CFD) methods. Lin et al. evaluated the boundary-layer separation control effect of the small vane-type vortex generators on the aerofoil in a landing configuration by wind tunnel test [7]. It was found that the vortex generator with a height of $0.18 \%$ aerofoil chord length can effectively reduce boundary layer separation and significantly increase the performance of the aerofoil. Gao [9] investigated the flow physics of VGs and how their sizes affect aerodynamic 
performance of a blunt trailing edge aerofoil DU97-W-300 using CFD simulations. Volino [10] studied the function of controlling boundary layer separation using the oscillating vortex generator jets situated on the suction side of a low-pressure turbine aerofoil. He found the jets were effective over a wide range of frequencies and amplitudes. Hibbs and Acharya [11] optimized the vortex generator geometry to enhance mass/heat transfer from the ribbed passage of a two-pass turbine blade coolant channel in an experimental study. Heffron et al. [12] compared three different mounting angles of MVG vane on the Eppler e387 aerofoil that was suffering flow separation and found that the MVG vane placed at $18^{\circ}$ was the most effective on flow control.

A pair of triangular MVGs with counter-rotating distribution was numerically investigated for the turbine aerofoil S809 by Yashodhar et al. [13]. In comparison to the unmodified case, the installation of MVGs was found to be able to continuously increase the skin friction and thus can suppress flow separation. The aerodynamic effect of VGs of six configurations on the wing of the RAF Javelin fighter was investigated by Paiboolsirichit using numerical method [14]. The results indicate that the VG could enhance wing's maximum lift and stall angle. The effect of the variables of VGs including installed angle, height and length were discussed and it was found that the installed angle affected the performance of VGs significantly. Similar result was obtained by Barrett and Farokhi [15]. They carried out wind tests to determine the performance of a two-dimensional wing section equipped with smart vortex generators (SVG) with the self-control device.

The optimum position and configuration of the MVGs on an unmanned aerial vehicle UAV wing was studied numerically by Chavez et al. [16]. It was found that the MVGs situated on the position after the detachment flow in the unmodified model provided the best effect on stall delay and the optimum height of the MVG is the height of the boundary layer. The effect of passive VGs on the UAV were investigated by Zhen et al. by both experiment and numerical method [17]. It was found that the VGs provided positive effect on the performance of the UAV by increase the maximum lift and the rectangular and curve-edge VG performs better than triangular VG.

The conventional geometry of VGs is a form of vanes on the suction side of an aerofoil near its leading edge. The VGs have different array configurations in terms of orientation as shown in Figure 1: 1) the counter rotating configuration, and 2) the corotating configuration. The counter rotating configuration is characterized by adjacent VGs having equal, but opposite installed angles to the flow. While the co-rotating configuration is characterized by adjacent VGs having all equal installed angles to the flow [18]. In Figure 1, the height of the vane is denoted by h, the length by e and the installed angle by $\beta$.

VGs are usually characterized by its height as relative to the thickness of boundary layer $\delta$. A typical vane-type VG has a similar height of the boundary layer. A higher VG can produce extra drag, which could compromise its aerodynamic benefit. Some experiments have shown that VGs lower than the boundary layer thickness can also introduce enough energy to the boundary layer with a relatively smaller drag increase. These VGs are effective enough in flow separation control. The sub- $\delta$-scale VGs that 

are shorter than $\delta / 2$ are referred to as micro-vortex generators (MVGs) [19]. The height of the MVGs adopted in the current work is about $0.2 \delta$.

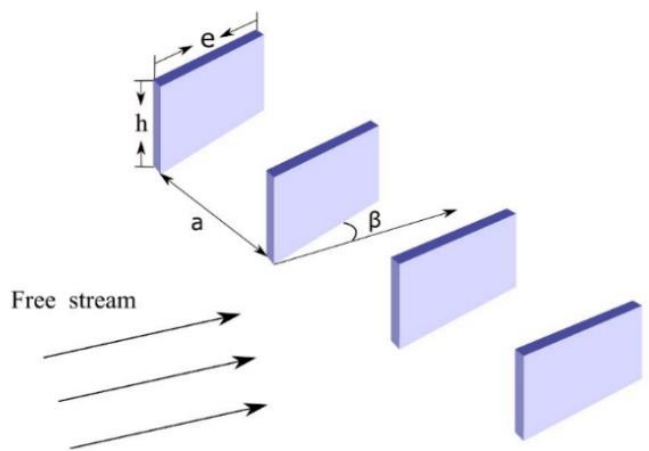

(a)

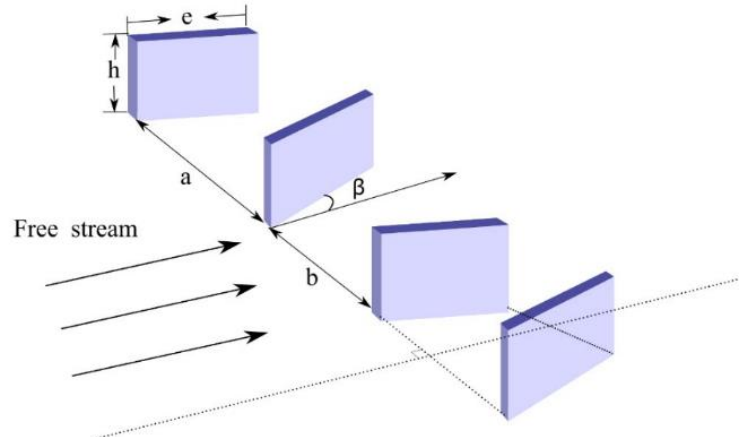

(b)

Figure1: Vortex generator configurations: (a) Co-rotating and (b) Counter-rotating

The main objective of this work is to find the best performing configuration of MVGs for an isolated aerofoil and a small-scale vertical axis wind turbine. The optimization of MVGs usually needs many experiments which are expensive. Using the Computational Fluid Dynamics codes Code_Saturne and Ansys-Fluent, the present work aims to determine the optimal variables of MVGs including installed angle, location and configuration, and investigate their aerodynamic effects on the turbines.

\section{GEOMETRY AND CASE SETUP}

\subsection{A single Micro-Vortex Generator on the plane}

In order to understand the flow control's effect of MVGs and carry out the code validation, a single MVG perpendicularly installed on a flat plane is investigated first. The computational domain and mesh distribution on the wall surface are shown in Figure 2. The installed angle is set at $16^{\circ}$ and the free stream velocity is $34.0 \mathrm{~m} / \mathrm{s}$. The MVG has a height of $7 \mathrm{~mm}$ and a length of $49 \mathrm{~mm}$. It is installed at the position where the thickness of the boundary layer is about $35 \mathrm{~mm}$. The length of the computational domain is about $4 \mathrm{~m}$, which is nearly 1000 times of the length of the MVG. The total number of hexahedron cells are 2.34 million. The boundary conditions are labeled in Figure 2 as inlet, outlet, symmetry and non-slip wall. The inlet boundary is defined based on the free stream velocity $34 \mathrm{~m} / \mathrm{s}$. The downwind outlet is defined as pressure outlet, where static pressure is specified.
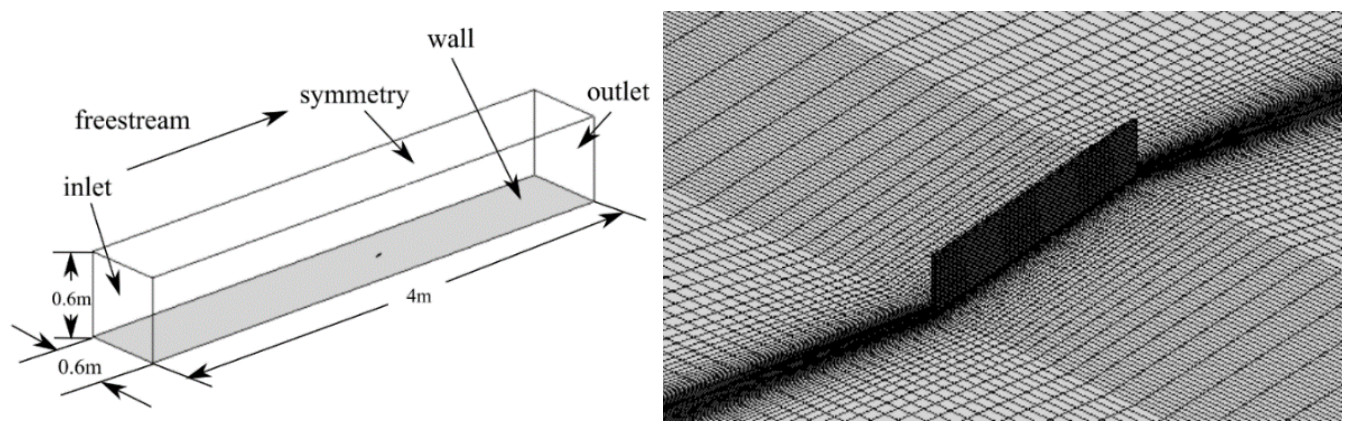

Figure 2: Geometry and mesh in the local region around VGs 


\subsection{MVGs on a single aerofoil}

Micro-Vortex Generators on the NACA0018 aerofoil were studied by unsteady Reynolds-averaged Navier-Stokes (URANS) method and large eddy simulations (LES). Both methods are detailed in section 3. This NACA 0018 profile is typical for VAWTs. Figure 3 illustrates the geometry of the aerofoil section equipped with one pair of MVGs of rectangular and triangular shapes with counter rotating configuration.

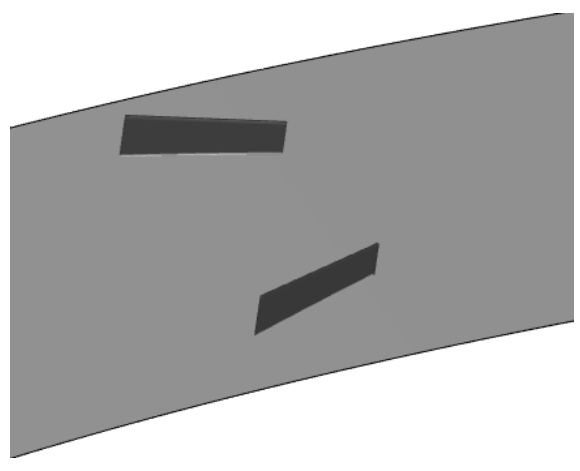

(a)

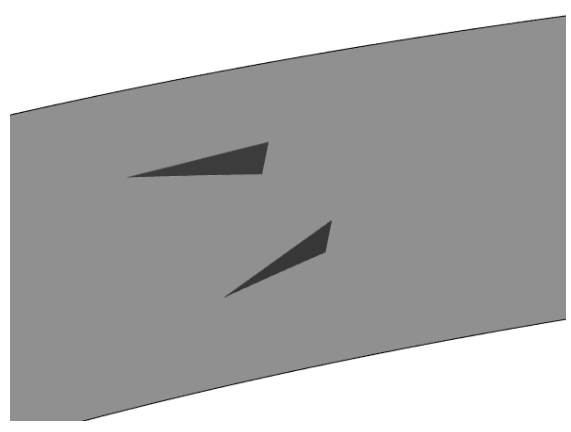

(b)

Figure 3: (a) Aerofoil with rectangular MVGs. (b) Aerofoil with triangular MVGs.

Optimization of MVGs has been discussed by several authors with the consideration of the variables including chordwise location, installed angle and length [20]. The study by Mueller-Vahl et al. shows that the MVGs located at $15 \%$ to $20 \%$ chord length from the leading edge of the aerofoil is ideal to realize the stall delay [21]. The wind tunnel test by Ashill indicates that the low-profile VGs set an angle of about $16^{\circ}$ is effective in flow separation control [22]. Therefore, Table 1 presents eight MVG models of various geometric parameters and among these MVGs, model A is regarded as the benchmark model.

Table 1 Tested MVG Models on the Aerofoil

\begin{tabular}{|c|c|c|c|c|c|}
\hline Test case & Configuration & Shape & Position & Angle $(\beta)$ & e/h \\
\hline A & Counter-rotating & Rectangle & $20 \% \mathrm{c}$ & $16^{\circ}$ & 3 \\
\hline B & Counter-rotating & Rectangle & $20 \% \mathrm{c}$ & $19^{\circ}$ & 3 \\
\hline C & Counter-rotating & Rectangle & $20 \% \mathrm{c}$ & $22^{\circ}$ & 3 \\
\hline D & Counter-rotating & Rectengle & $15 \% \mathrm{c}$ & $16^{\circ}$ & 3 \\
\hline E & Counter-rotating & Rectangle & $22 \% \mathrm{c}$ & $16^{\circ}$ & 3 \\
\hline F & Counter-rotating & Rectangle & $25 \% \mathrm{c}$ & $16^{\circ}$ & 3 \\
\hline G & Counter-rotating & Triangle & $20 \% \mathrm{c}$ & $16^{\circ}$ & 3 \\
\hline H & Counter-rotating & Rectangle & $20 \% \mathrm{c}$ & $16^{\circ}$ & 6 \\
\hline
\end{tabular}

The chord length of the aerofoil is $0.246 \mathrm{~m}$ and the computational domain spanwise length is about $30 \%$ of the chord length. The free stream velocity is $10 \mathrm{~m} / \mathrm{s}$ and the Reynolds number based on the aerofoil chord length is $1.6 \times 10^{5}$. In all models, the height of the MVGs was about $1 \%$ of the aerofoil chord length. The pitch spacing 
between the adjacent MVGs is three times of its height in order to eliminate the influence between each other.

The common C-H type mesh was adopted as Figure 4. The Farfield boundary was located 40 times of chord length away from the aerofoil. Velocity INLET and pressure OUTLET boundary conditions were applied at the inlet and outlet domain, respectively. The aerofoil and MVGs were set as non slip walls. A periodic condition is enforced at the spanwise direction. The structured grid was deployed in the whole domain. There were 300 points along the surface of the aerofoil.

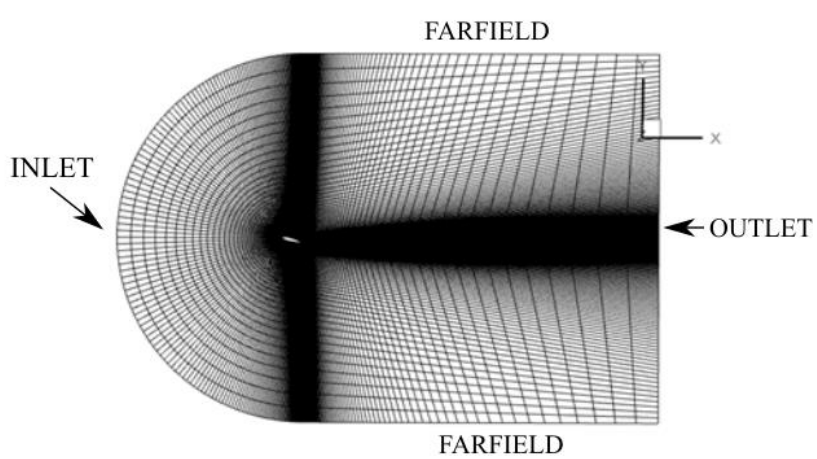

Figure 4: C-H type computational domain

\subsection{VAWT with VGs}

After the validation and flow study of the isolated aerofoil, an H-type Darrieus vertical wind turbine will be investigated. The schematic view of this turbine is given in Figure 5. This wind turbine consists of three vertical blades, one vertical support and six horizontal struts.

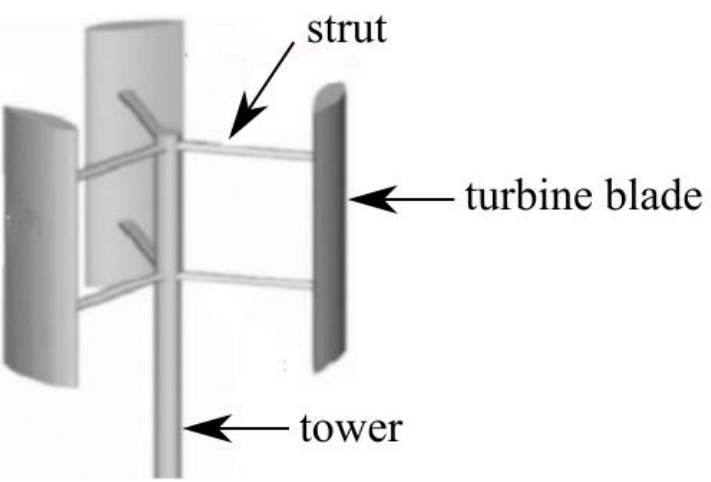

Figure 5: H-type vertical axis wind turbine

The geometry of the computational domain and the boundary conditions are given in Figure 6(a). To have high-quality meshing, struts are not included in the current computational domain. As the rotor is a moving surface, the whole computational domain was divided into two sub-domains (ROTOR and STATOR domains) with an interface between them. The ROTOR domain is a circular inner zone that includes the wind turbine. This ROTOR domain rotates at a fixed angular velocity. The STATOR domain is a large stationary rectangular domain outside the inner zone. The mesh cells on both sides of the interface have the same size to achieve a smooth and sliding transition. 
This wind turbine blade is the NACA 0018 aerofoil that was discussed in the last section, which can provide high lift-to-drag ratio. The main turbine parameters are given in Table 2.

Table 2 Rotor Parameters

\begin{tabular}{|c|c|}
\hline Number of blades & 3 \\
\hline Blades aerofoil & NACA 0018 \\
\hline Blade chord(c) $[\mathrm{m}]$ & 0.246 \\
\hline Radius $(\mathrm{R})[\mathrm{m}]$ & 0.85 \\
\hline Wind speed $(\mathrm{V})[\mathrm{m} / \mathrm{s}]$ & 8 \\
\hline Tip speed ratio & $1-3.5$ \\
\hline Height of blades $(\mathrm{H})[\mathrm{m}]$ & 0.08 \\
\hline
\end{tabular}

The turbine is assumed to operate in an open field. To avoid wall blockage, the length and width of the STATOR domain are 40R and 10R respectively. The radius of the ROTOR zone is 1.2 times of the turbine radius. Figure 6(b) shows a zoom-in view of the mesh around the turbine blades. The inlet boundary was set at a constant wind speed of $8 \mathrm{~m} / \mathrm{s}$, while the atmospheric pressure boundary was imposed at the outlet. The symmetry boundary condition was adopted for the top and bottom boundaries in Figure (6a) and the periodic boundary conditions were assumed in the spanwise direction. Noslip wall boundary condition is implemented on the blade and MVG surface.

The turbine operated with a fixed wind speed (V), whereas the rotational speed of the turbine $(\omega)$ changes to achieve different tip speed ratios. The Tip Speed Ratio (TSR) is defined as $\lambda=\mathrm{R} \omega / \mathrm{V}$ (V stands for the wind velocity).

The simulation is regarded to be fully developed if the instantaneous moment coefficient of the turbine was less than $1 \%$ different compared to the value of the same azimuth angle of last period. For the LES calculations, the flow becomes fully developed after about 10 revolutions, and then, the phase averageing was performed for the following five revolutions.

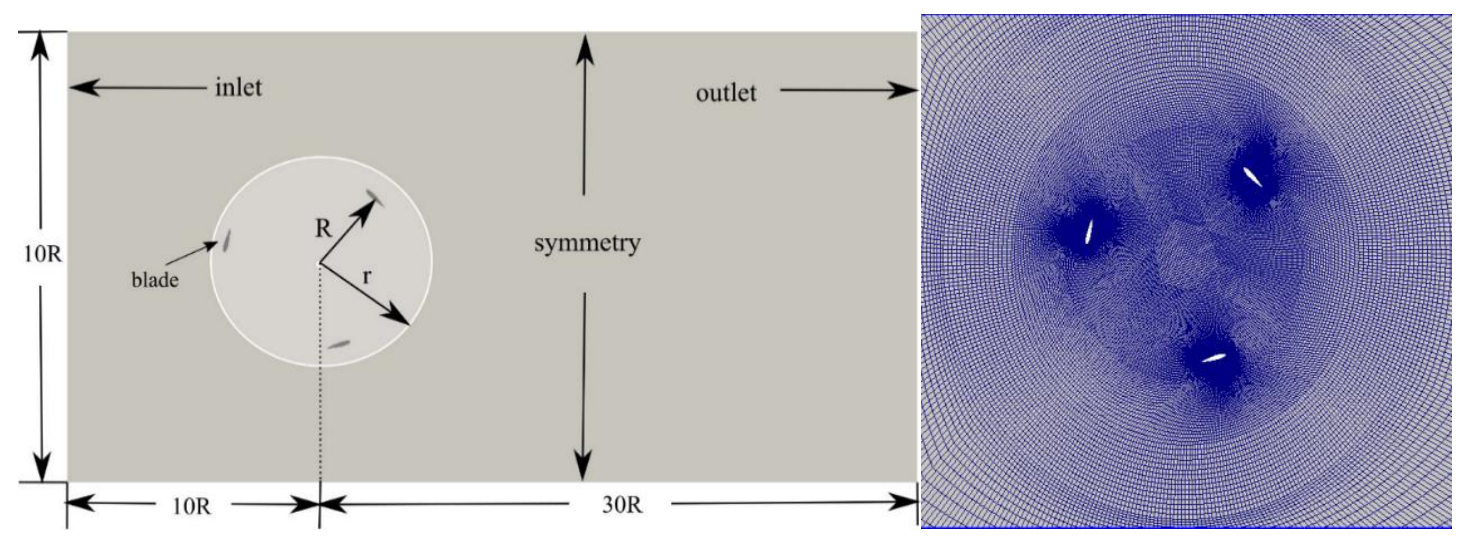

(a)

(b)

Figure 6: (a) competational domain (b) mesh in the local region around turbine blades 


\section{NUMERICAL METHOD}

Code_Saturne and ANSYS-Fluent were used for the CFD calculations in this study. Code_Saturne of EDF is a general-purpose open source CFD software package based on the finite volume method and a cell-centered approach. The LES simulations were performed by Code_Saturne in the current work, whereas the ANSYS Fluent simulation package was used for the (U)RANS calculations.

For the unsteady RANS Fluent calculation, the well-known two-equations SST (Shear Stress Transport) k- $\omega$ turbulence model proposed by Willcox [23] was chosen. This method attempts to predict turbulence by solving two equations for the extra two variables, turbulent kinetic energy $(k)$ and specific dissipation rate $(\omega)$. It blends the $\mathrm{k}$ $\omega$ model and the k- $\varepsilon$ model, which performs better for wall-bounded cases, especially under the adverse pressure gradients [24]. The pressure-based solver with the second order spatial scheme and the SIMPLE time marching method were adopoted. No wall function was applied as the mesh resolution near the wall is fine enough.

The LES calculations were performed by Code_Saturne, an unstructured, collocated finite-volume code. All large eddy simulations were carried out with the second order central difference scheme in space and time. The implicit LES (ILES) is adopted for the current study. It uses the numerical dissipation as a subgrid model [25], and thus, no subgrid scale model is imposed. Recently, there has been an increasing interest in ILES approach and its effectiveness has been demonstrated in a wide range of applications for various fields from fluid engineering to astrophysical fluids computations [26].

\section{RESULT AND ANALYSIS}

\subsection{A single MVG on the plane}

The simulation of a single MVG installed on a flat plane has been compared with the experimental results, as shown in Figure 7. Six streamwise stations behind the trailing edge of the MVG are given, which are $s / h=10,17,50$ and 109. Here, $s$ is the distance between the station and the trailing edge of MVG. The column (a) in Figure 7 and Figure 8 present the experimental results from Yao et al. [27]. The experiment were conducted in the Langley 20- by 28 -Inch Shear Flow Tunnel. The free-stream velocity is $34 \mathrm{~m} / \mathrm{s}$. A $12.7-\mathrm{mm}$ thick splitter plate was used to eliminate any upstream influence. A single VG was located approximately $2.25 \mathrm{~m}$ downstream of the boundary layer trip where the boundary-layer thickness $(\delta)$ was approximately $35 \mathrm{~mm}$. The column (b) show the CFD results of RANS from Fluent. The present numerical study was conducted in the same conditions with the experiment in the literature by Yao et al. [27]

As can be seen in Figure 7, the vortex development downstream of the trailing edge of MVG from the numerical calculations agrees qualitatively well with the measurement data. Figure 7 shows the contour of the streamwise velocity at measurement stations from RANS. As the vortex moves downstream from the generator, the size of vortex increases, but the intensity diminishes and the vortex core moves away from the flat plate. The transparent square in the figure denotes the spanwise location of the vortex generator. It can also be observed that the vortex core moves away from the spanwise location of the MVG when it travels downstream. 

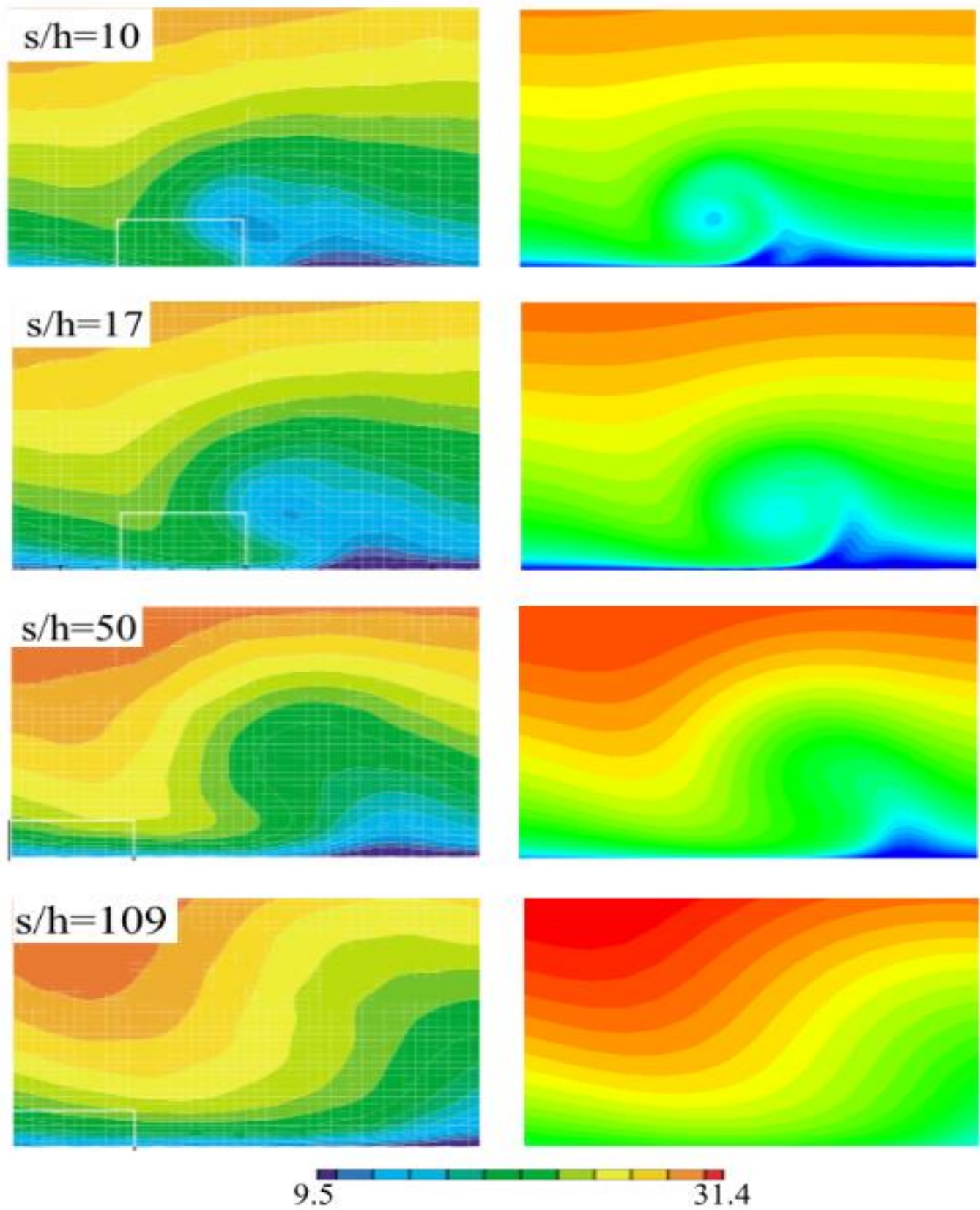

(a)

(b)

Figure 7: Comparison of streawise velocity at different streamwise stations (a) Experiment [27] (b) RANS

Figure 8 portrays the contour of the streamwise vorticity at different sections. As $\mathrm{s} / \mathrm{h}$ increases, the magnitude of the streamwise vorticity decreases and at section $s / h=109$, the vortex has been fully diffused. This demonstrates the streamwise length in which the MVG can be effective, pointing to the need to carefully choose the location of MVG.

Figure 9 shows the comparison of between the numerical result in present work and the experimental data and CFD result from the literature by Yao [27] in terms of the variation of half-life radius of vortex. The unsteady RANS of k- $\omega$ SST model was used in both CFD studies. The half-life radius is defined as the distance between the center of the vortex core and the position where the vorticity was equal to half of the peak 
vorticity. It was found that the half-life radius increases almost linearly with $\mathrm{s}$ and the curves of numerical results have the same trend with experimental data. The URANS result agrees well with each other in both CFD studies. The CFD method overestimates the half-life readius by about $38 \%$ at $\mathrm{s} / \mathrm{h}=10$. As the vortex is not exactly cycle, the measurement errors are difficult to avoid. In addition, the difference is raleted to the application of turbulence model.
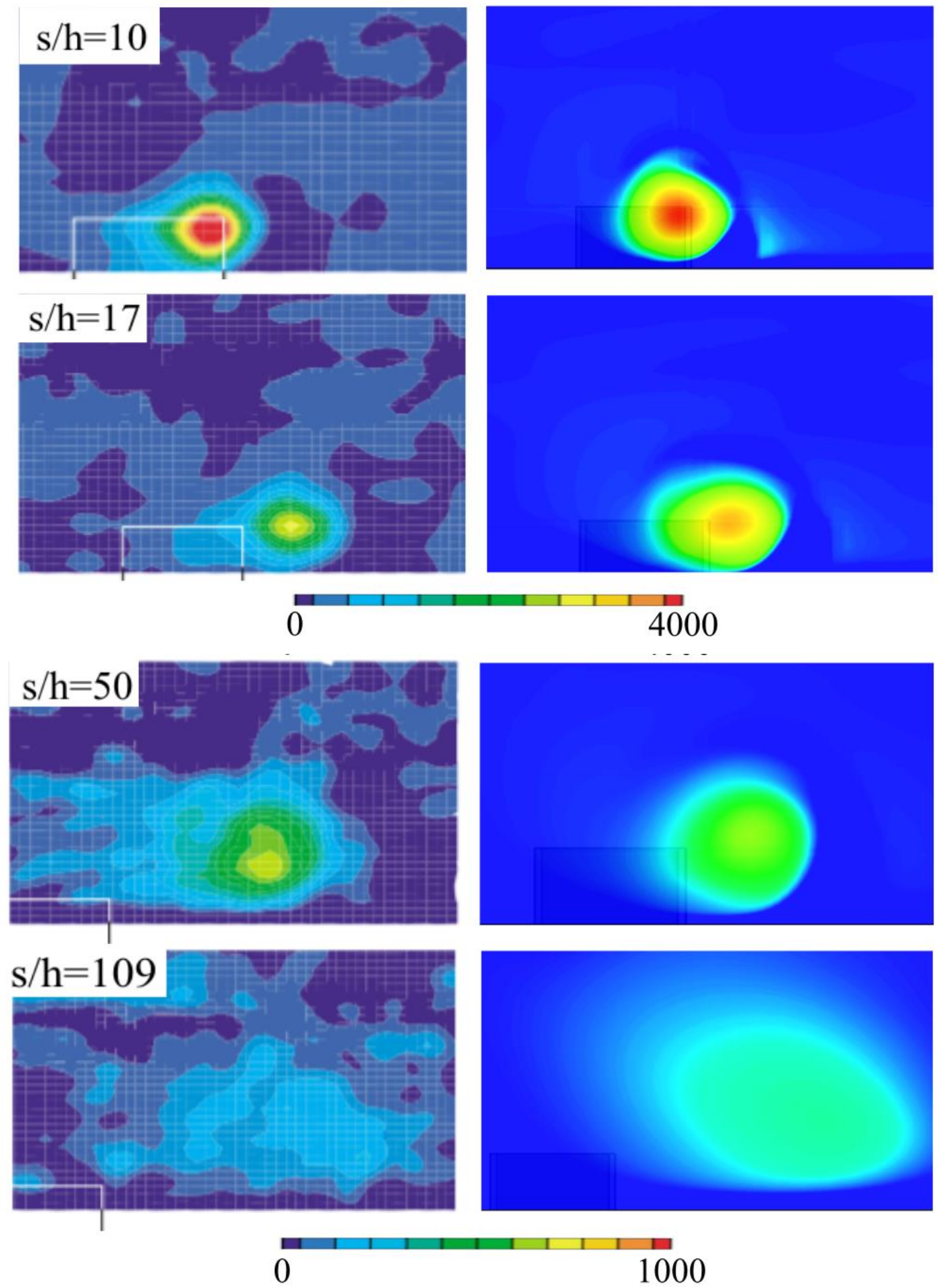

(a)

(b)

Figure 8: Comparison of streamwise vorticity at different streamwise stations (a) Experiment [27] (b) URANS 
304

305

306

307

308

309

310

311

312

313

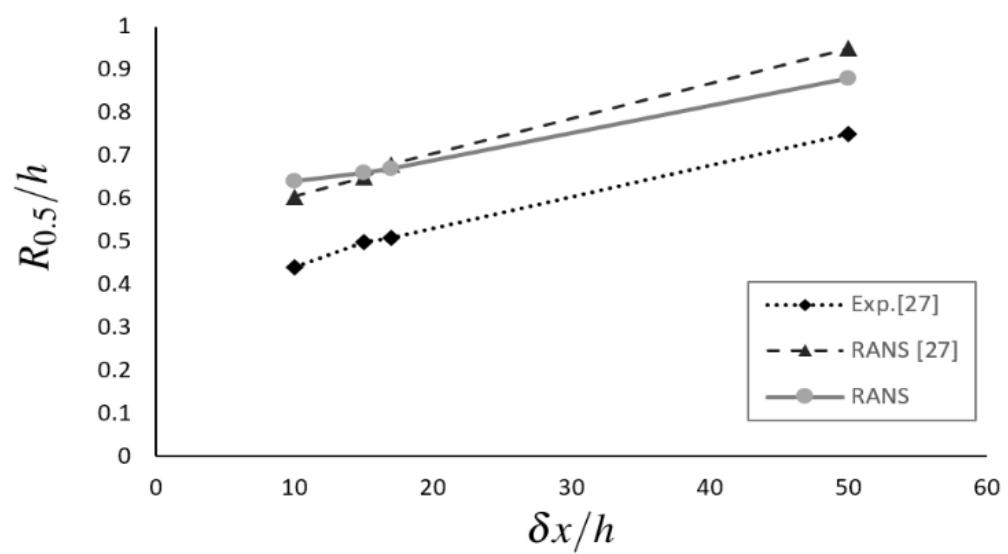

Figure 9: Vortex half-life radius nondimensionalized by device height.

\subsection{Aerofoil with MVGs}

\subsubsection{Baseline and Mesh Sensitivity}

In the clean aerofoil case, the typical feature of its flow field can be seen from a side view of the iso-surfaces of $\mathrm{Q}$ colored by velocity magnitude at $R e_{c}=1.6 \times 10^{5}$ as in Figure 10. The flow features a laminar separation bubble near the leading edge of the aerofoil, a transition to turbulence immediately after the laminar separation, a flow reattachment of the shear layer and turbulent separation can be seen when the aerofoil is placed at a high angle of attack (AoA).

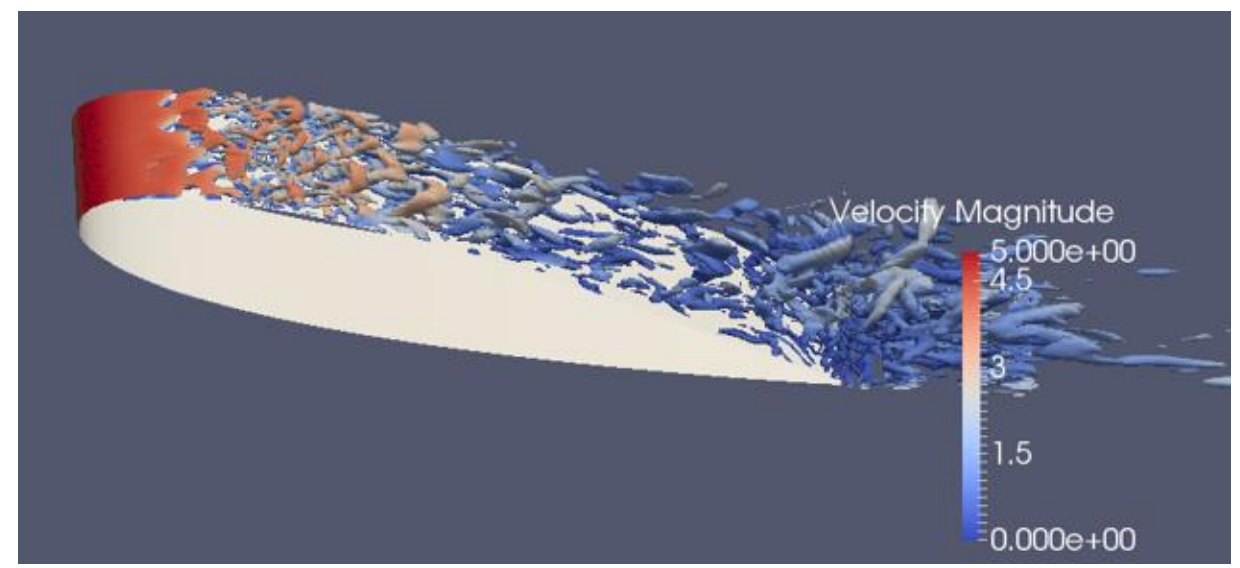

Figure 10: Iso-surfaces of $Q$ colored by velocity magnitude for the case of clean aerofoil NACA 0018, $\mathrm{Q}=1000$, AoA $=14^{\circ}$, LES.

In order to verify the validity of the study, a baseline of three dimensional NACA 0018 aerofoil was carried out to establish the sensitivity of the simulation to the mesh revolution. Three different meshes with various height of first grid cells near the wall were tested compared to the experimental results of Sheldahl et al. [28] in terms of the time averaged lift and drag coefficient as shown in Table 3. Convergence towards the experimental results is clearly seen as the number of grid cells is increased. The difference in $C_{L}$ between Mesh 2 and the experimental value is only about 2.0\%, while the difference in $C_{D}$ is $4.1 \%$, Further increase of the mesh size to Mesh 1 yielded a small change and hence Mesh 2 was chosen. 
Table 3 Comparison of RANS result and experimental data of 3D NACA 0018 aerofoil in terms of lift and drag coefficient, $\mathrm{AoA}=13^{\circ}$

\begin{tabular}{|c|c|c|c|c|}
\hline & Total Cells & $C_{L}$ & $C_{D}$ & $C_{L} / C_{D}$ \\
\hline Sheldahl et al. [28] & & 0.950 & 0.0545 & 17.43 \\
\hline Mesh 1 & $8.43 \times 10^{6}$ & 0.937 & 0.0538 & 17.19 \\
\hline Mesh 2 & $4.79 \times 10^{6}$ & 0.932 & 0.0524 & 17.78 \\
\hline Mesh 3 & $1.38 \times 10^{6}$ & 0.911 & 0.0472 & 19.3 \\
\hline
\end{tabular}

Figure 11 shows the lift and drag coefficients variation with the angle of attack $(\alpha)$. As shown in the figure, the lift coefficient of the clean aerofoil from RANS results agrees well with the experiments. For the drag coefficient, the CFD data matches well with the experiment before the stall occurs. After that, the drag coefficient from the numerical result is smaller than the experimental result. This difference is also reported in other studies [5], which is mainly due to the turbulence model limitation for the separated flow. Figure 11 indicates a good agreement between numerical result and measured data in terms of lift-to- drag ratio.

In the case with MVGs (case A), it can be seen that the MVGs can improve the aerodynamic performance of the aerofoil significantly. At a very small angle of attack, the lift coefficient of the MVG case is close to that of the clean aerofoil. As the angle of attack increases to around $14^{\circ}$, the stall occurs in the clean aerofoil case with the lift rapidly drops. However, the lift on the aerofoil installed with the MVGs still increases until the angles of attack reached $16.5^{\circ}$. It is evident that the MVGs can increase the stall angle as well as the maximum lift coefficient.

For the drag coefficient, a slightly higher drag is observed in the MVG case as compared to the clean aerofoil before the stall. This is due to the fact that the vortex generator does nothing but to slightly increase the skin drag for the attached boundary layer. As the angle of attack increases beyond the stall angle, it is evident that the drag is significantly less for the aerofoil with MVGs installed. In addition, the positive effects of MVGs can be seen by the lift-to-drag ratio comparison between the cases with and without MVGs in Figure 12. At high angles of attack the aerofoil with MVGs has a relatively higher lift-to-drag ratio compared to the clean aerofoil case, but there is a small price to pay at low angles.
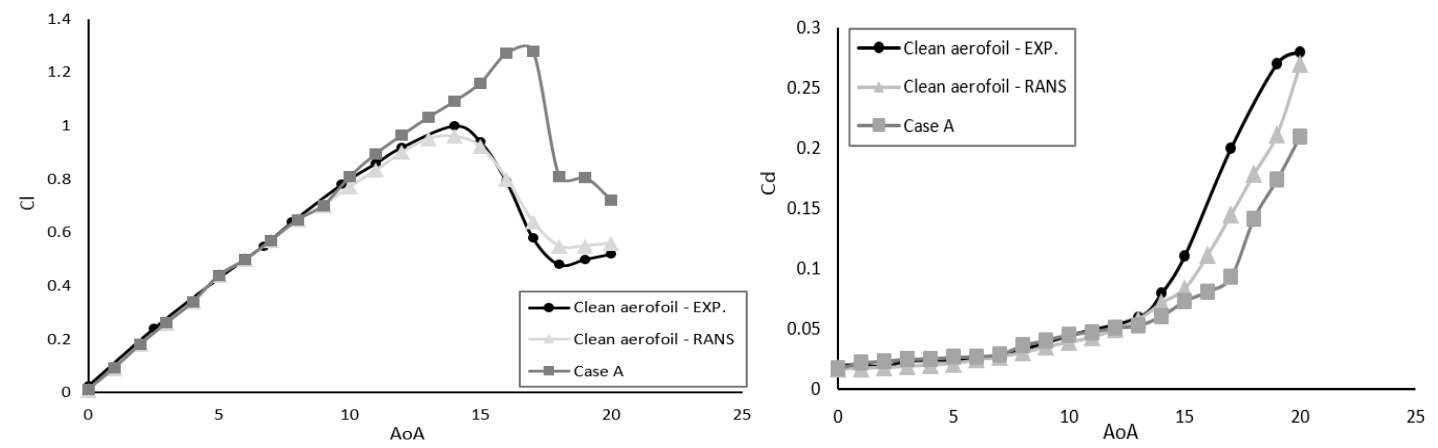

Figure 11: Aerofoil performance at different angles of attack: (a) lift coefficient and (b) drag coefficient, URANS [28]. 


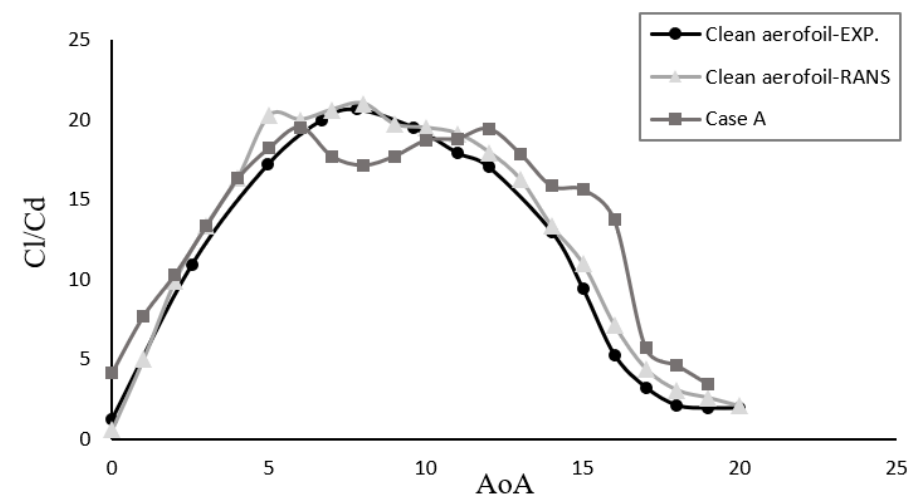

Figure 12: Lift-to-drag ratio comparison between aerofoils with and without MVGs, URANS [28].

Figure 13 shows a comparison of the mean value of pressure coefficient $(C p)$ at $\mathrm{AoA}=15^{\circ}$ for the aerofoil with and without MVGs. As can be seen from the figure, $C p$ on the suction side of the aerofoil is improved after adding the MVGs. As the result, the pressure difference between the suction and pressure side of aerofoil is increased, leading to the higher lift.

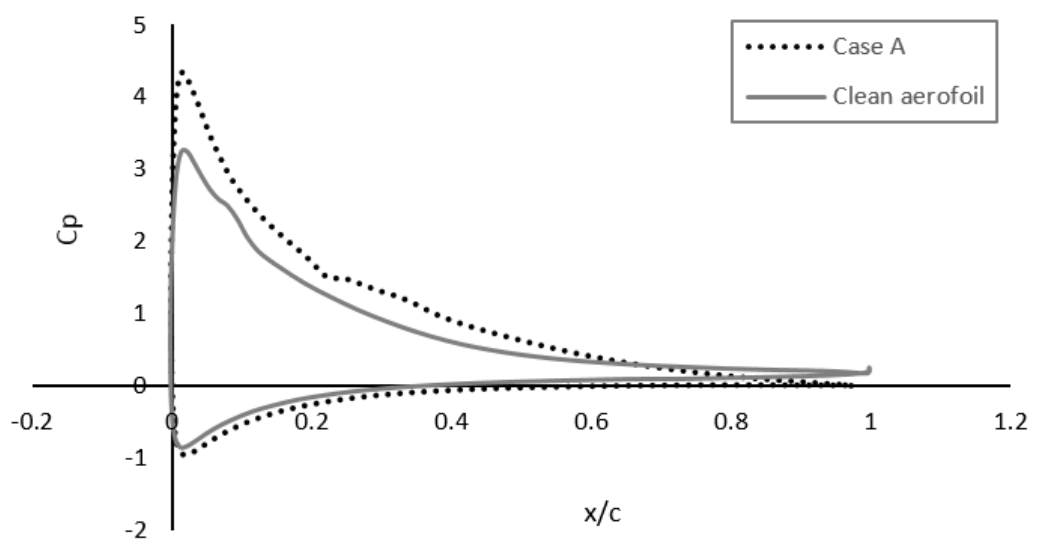

Figure 13: Pressure coefficient comparison between aerofoils with and without MVGs, $A o A=15^{\circ}$, URANS.

Wall shear stress is a useful parameter to assess the effect that the vortices have on the near-wall boundary layer. Figure 13 presents a comparison of the skin friction coefficient along the upper surface of aerofoils with and without MVGs at a high angle of attack $15^{\circ}$. The solid line shows the $C_{f}$ distribution of the clean aerofoil. $C_{f}$ drops sharply near the leading edge at about $5 \%$ chord length caused by the small leadingedge bubble. The value of $C_{f}$ increases, as the flow reattaches. Further downstream a turning point appears at about $15 \%$ chord length of aerofoil where $C_{f}$ starts decreasing again leading to very low values at $x>0.4 \mathrm{c}$ due to massive flow separation.

The dashed line in Figure 14 stands for the aerofoil of case A. Near the trailing edge of the aerofoil, the trend of $C_{f}$ distribution of case $\mathrm{A}$ is close to the clean aerofoil. However, there is a sudden rise in $C_{f}$ at $25 \%$ chord length just downstream of the MVGs. Further downstream $C_{f}$ increases again due to the flow transition from laminar to turbulence and reattachment. 


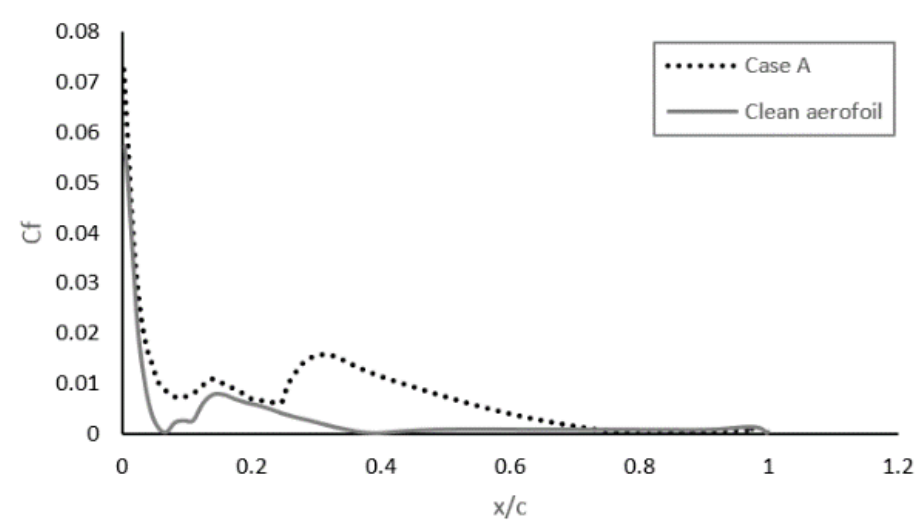

Figure 14: Skin friction coefficient distribution on NACA 0018, AoA $=15^{\circ}$, URANS.

As momentum is introduced into boundary layer by the MVGs, the distribution of the skin friction along the surface changes significantly. Figure 15 shows the skin friction at $\mathrm{s} / \mathrm{h}=3,5,10$ and 30 behind the MVGs where $\mathrm{s}$ stands for the distance to the trailing edge of the MVGs and $h$ is the height of MVGs. With MVGs on the aerofoil, a larger variation of skin friction is observed at $\mathrm{s} / \mathrm{h}=3$ compared to a clean aerofoil. The increased level of skin friction is an indication of a healthier boundary layer with no intention to separate. They can improve the skin friction on the wall surface of an aerofoil, which agrees well with other results [27]. This improvement was induced by the vortices behind the MVGs. Along the spanwise direction, the skin friction decreases with the increase of distance from MVGs. Along the chord line direction, skin friction near the MVGs is relatively higher than that farther from MVGs because of the diffusion of vortices.
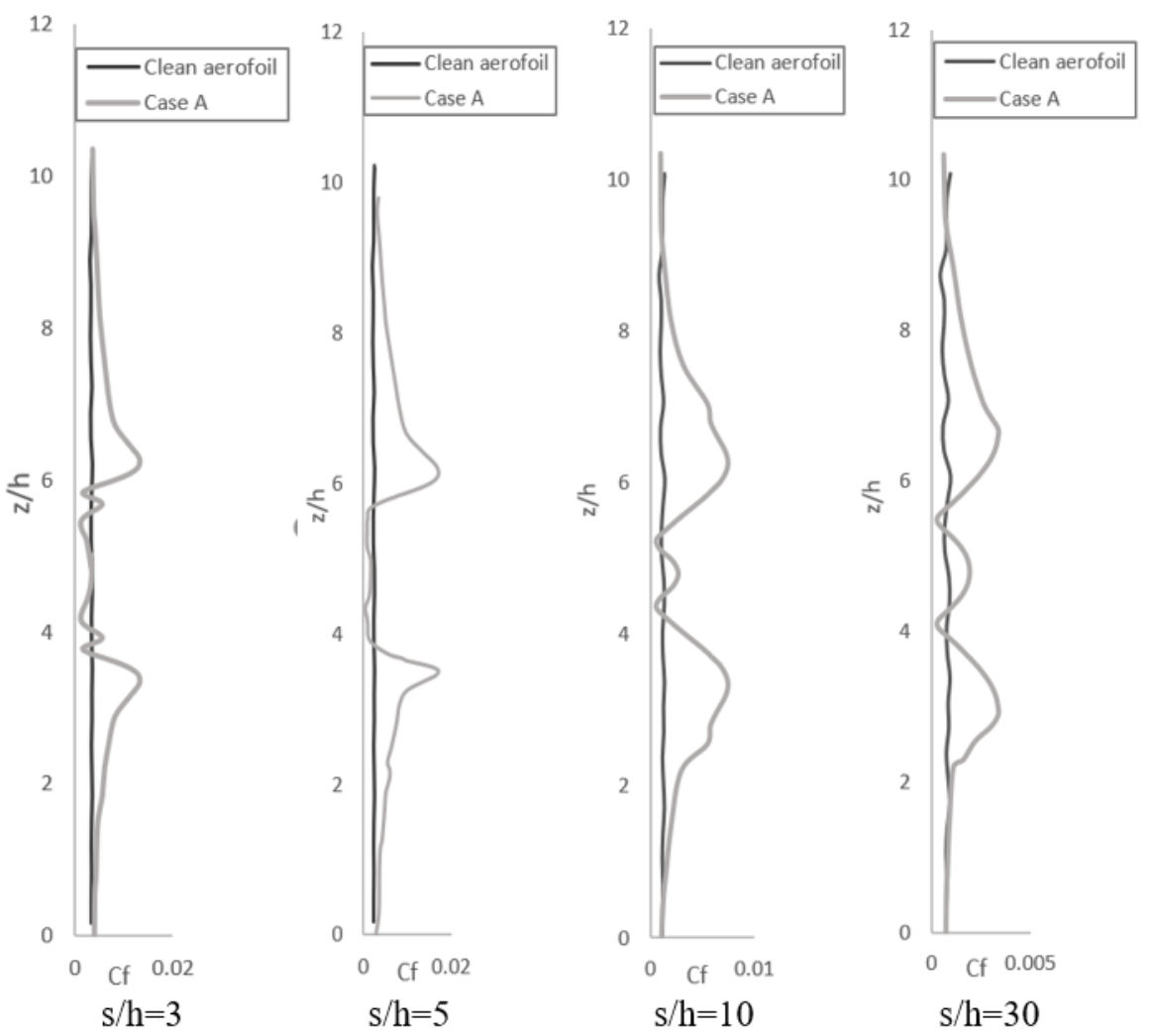

Figure 15: Skin friction coefficient distribution at different points on aerofoil surface, RANS. s stands for the distance to the trailing edge of MVGs. $\mathrm{h}$ is the height of MVGs and $\mathrm{z}$ is the coordinates in $\mathrm{Z}$ direction, URANS. 
MVGs with a proper configuration can have a positive effect on the aerodynamic performance of a NACA 0018 aerofoil. In order to optimize the MVG configuration for a better performance, a comprehensive understanding of the influence of several parameters related to MVGs is important, such as the location, installed angle, length, shape and array configurations.

\subsubsection{Effect of location on the performance of MVGs}

Many researchers have shown that the location of MVGs influences the capability of controlling flow separation. It was found that MVGs located at $15 \%$ to $30 \%$ of the chord length could improve the aerodynamic performance of the aerofoil. In the present work, besides case A with MVGs located at $20 \%$ chord length, three other cases were studied, in which the MVGs were located at 15\%,22\% and 25\% chord length. The lift and drag coefficients versus the angle of attack for these cases are given in Figure 15. The clean aerofoil case is also superimposed. Compared to the clean aerofoil case, cases A, D and E have significantly improved the lift near the stall angles of attack, especially in the case E, where the maximum lift has been improved by $25 \%$. However, the MVGs in case $\mathrm{F}$, which are located at $25 \%$ chord length of the aerofoil, have a negative effect on other aspects of aerodynamic performance. The stall angle and the lift after the stall have also been reduced. For the drag, all the cases with MVGs have a similar trend as discussed in the last section. Compared to the clean aerofoil, all four configurations with MVGs have a mildly higher drag at lower angle of attack. However, after the aerofoil stalled, a lower drag is observed in the MVGs cases. Among the cases tested, case A has the best overall performance where the highest lift and the lowest drag are observed.
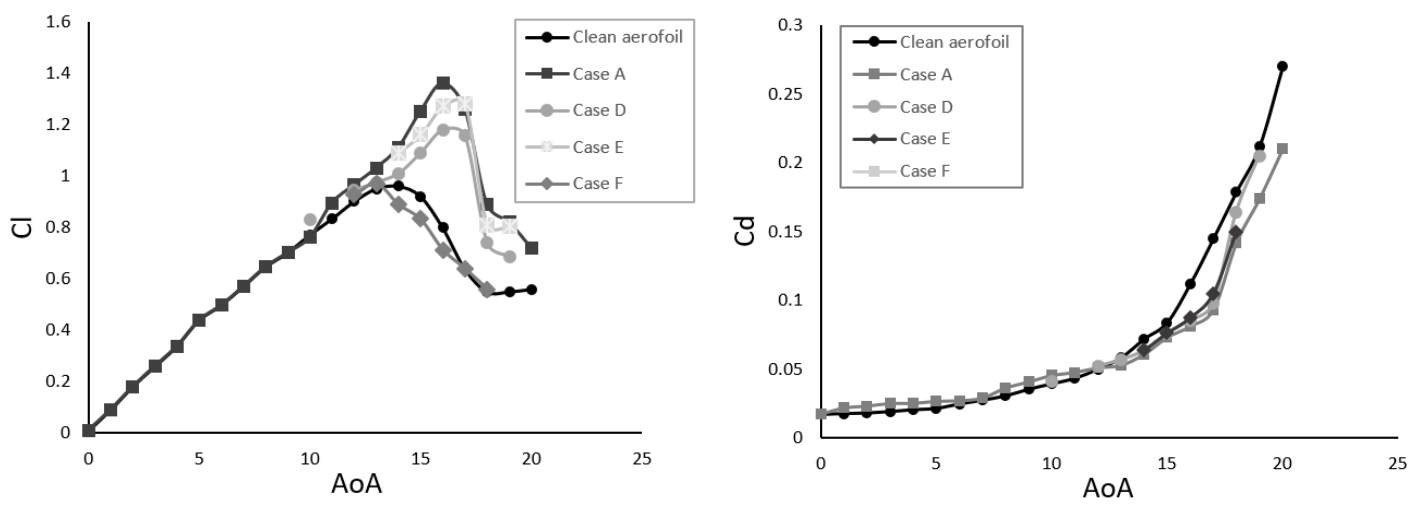

Figure 16: lift and drag coefficient comparison of different cases, URANS.

The contours of skin friction on the suction side are shown in Figure 17, where MVGs are installed in three different streamwise locations. The flow direction and the position of leading edge of the aerofoil are present in the figure. Compared to the clean aerofoil case, the MVGs increase the skin friction which indicates a healthier boundary layer. There is a region of high skin friction in cases A, D and E due to the generation of a pair of counter-rotating vortices behind the trailing edge of MVGs, see Figure 18 for illustration. This improvement is most evident in case A, where the MVGs are located at $20 \%$ chord length; whereas in case E, where the MVGs are located at $22 \%$ chord length, there is no noticeable region of high skin friction behind MVGs. 
A strong variation of the skin friction in the spanwise direction can be observed in Figure 15. To examine this variation, Figure 19 plots the skin coefficient for all the cases with MVGs installed. The data at the station downstream of the MVGs at $\mathrm{s} / \mathrm{h}=5$ is extracted. It is evident that the skin coefficient for the case A is highest among all the cases, which indicates that the strongest vortex is generated in case $\mathrm{A}$.

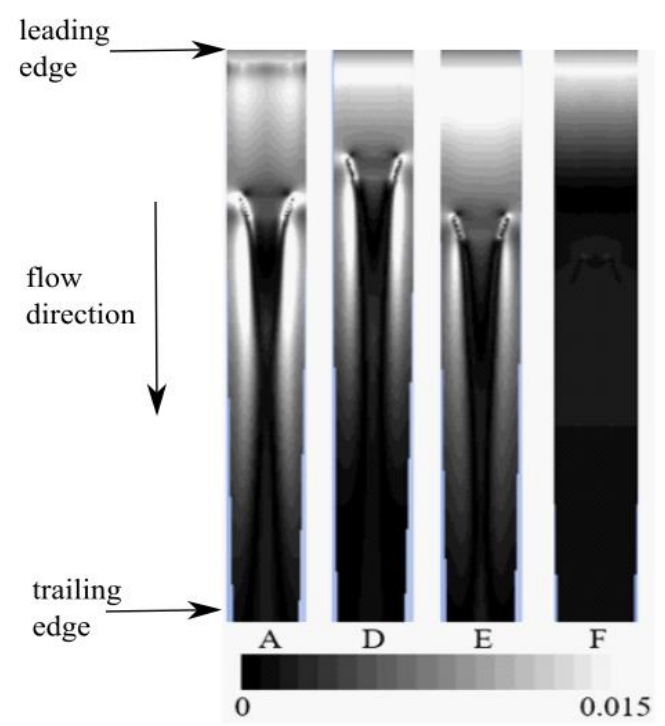

Figure 17: Skin friction coefficient contours on the aerofoil surface on the suction side, $\mathrm{AoA}=15^{\circ}$, URANS.

\section{region of increased skin friction}

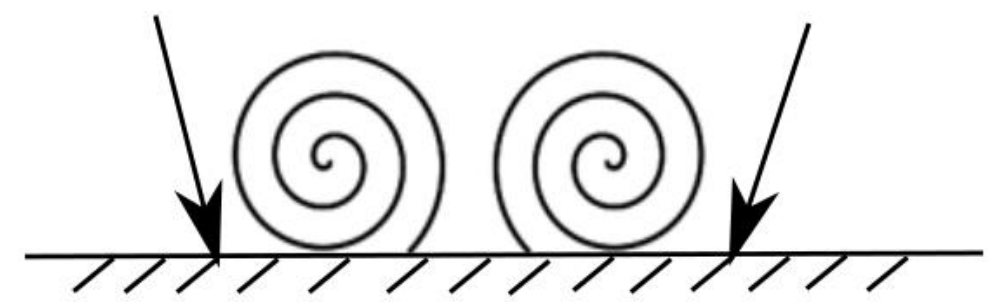

Figure 18: schematic view of one pair of vortices behind MVGs.

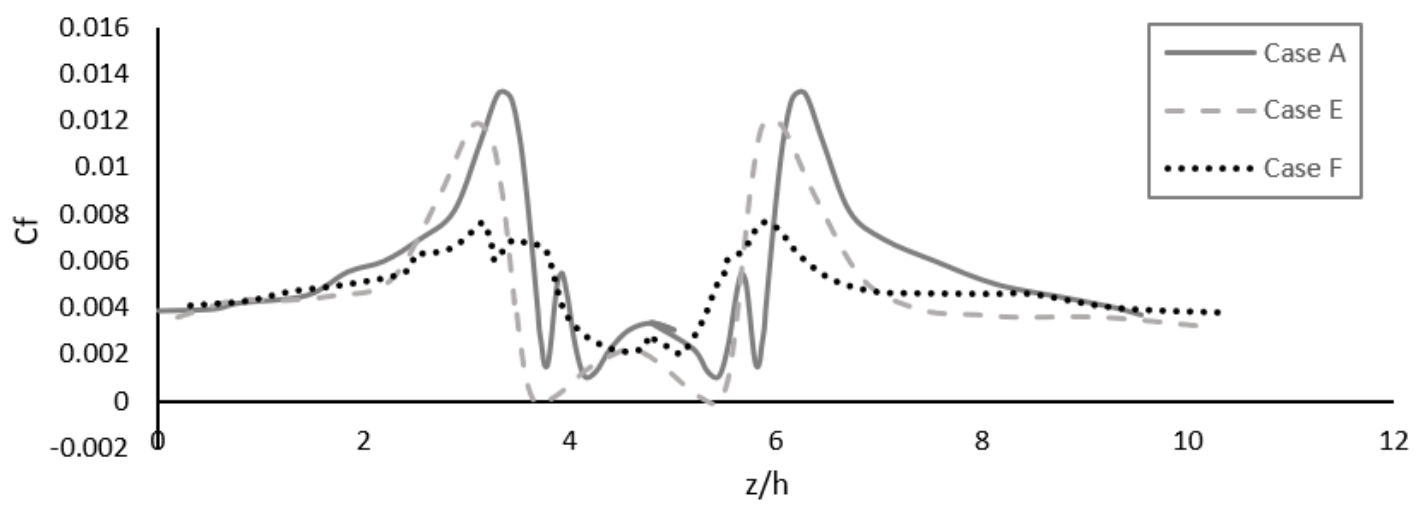

Figure 19: Skin friction selected streamwise section, .x/h $=5$, AoA $=15^{\circ}$, URANS.

Figure 20 shows the comparison of streamlines with and without MVGs at different locations at the angles of attack of $15^{\circ}$. From the clean aerofoil, the separation occurs at around half of chord length, pointing to a stall of the trailing edge separation process. 
In case $\mathrm{D}$, where the MVGs are installed at $15 \%$ chord length, there is a small separation bubble near the trailing edge on the suction side of aerofoil. When the MVG moves to the location of $20 \%$ chord length in case A, the flow stays attached over the whole suction side of the aerofoil. The MVGs in case $\mathrm{E}$ and case $\mathrm{F}$ are located $22 \%$ and $25 \%$ of the chord length respectively. It is clear that in case $\mathrm{F}$ the area of the separation region significantly increases in the aft-portion of the chord, with the size of the trailing edge separation bubble being the largest.
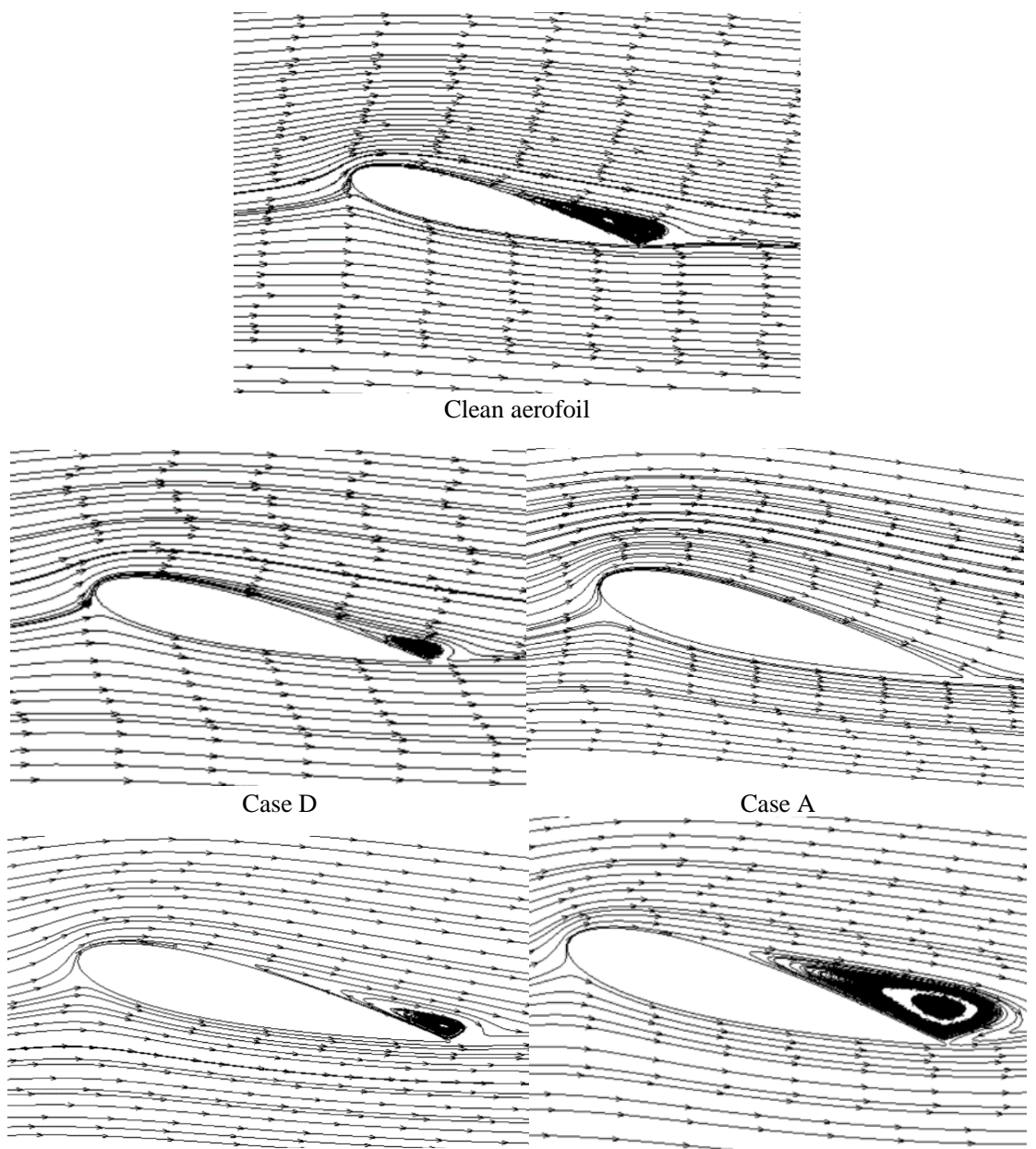

Case E

Case F

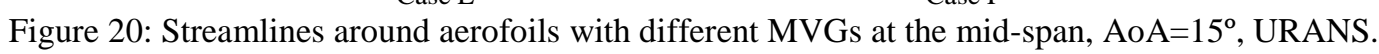

\subsubsection{Effect of installed angle on the performance of MVGs}

Apart from the location, the installed angle is also of great importance for the performance of MVGs. The MVGs of a larger installed angle introduce more energy into the boundary layer. However, they may introduce higher drag at smaller angle of attack, which may offset the benefit of the separation control. As a result, finding an optimal installed angle to balance the lift and drag increases is essential.

The comparison of the lift and drag for the aerofoils with MVGs installed at three different angles is shown in Figure 21. Like other cases discussed above, MVGs have no visible effect on the lift at small angles of attack, while the drag is slightly increased. The lift coefficient continues to increase and peaks at 1.3 in cases $\mathrm{A}$ and $\mathrm{B}$, while the 
clean aerofoil has already stalled. The drag in case A follows the same trend with case $\mathrm{B}$, which is slightly smaller than the clean aerofoil after stall angle.
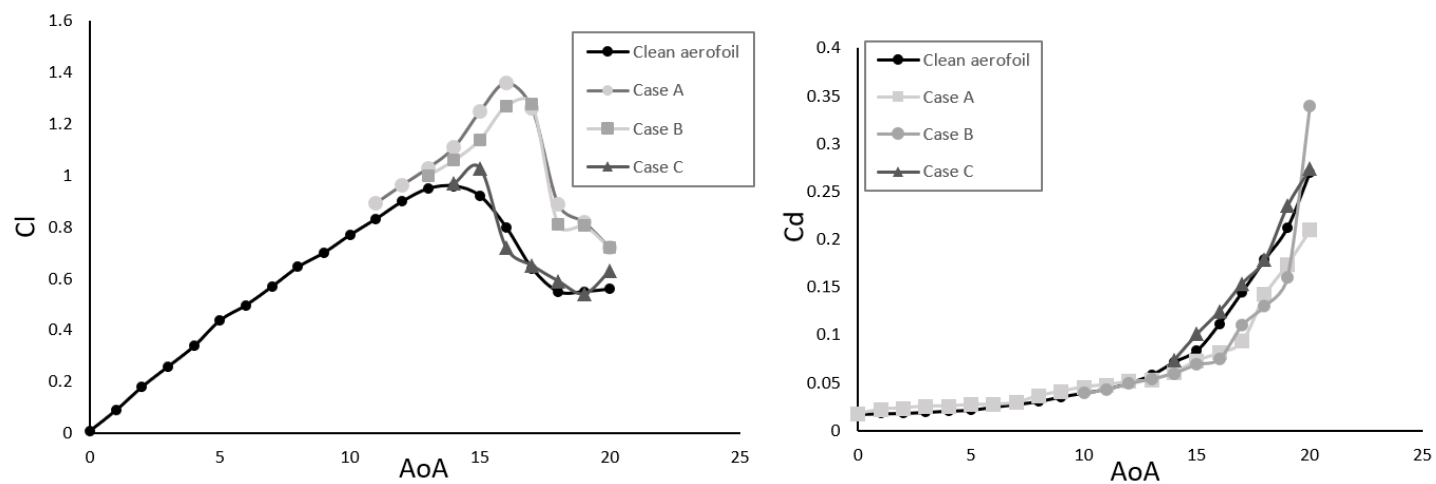

Figure 21: lift (right) and drag (left) coefficient comparison of different cases, URANS.

As discussed before, the suppression of the separation bubble by the MVGs can be shown by the contours of the skin friction on the suction surface of the aerofoil, which are shown in Figure 22. The spanwise distribution of the skin friction coefficient is shown in Figure 23 at $\mathrm{s} / \mathrm{h}=4$. The skin friction coefficient is extracted from the location $\mathrm{s} \mathrm{m}$ downstream the MVGs. The skin friction increase can be observed both in case A and B, but not in case C. Figure 22 shows the contour of skin friction coefficient distribution. There is a region of high skin friction downstream of the MVGs in these two cases, which corresponds to the result of the lift enhancement showed in Figure 21. However, in case $\mathrm{C}$, as the installed angle of the MVGs is too high, the counter rotating vortex is not strong enough to suppress the separation bubble, and thus, the skin coefficient is similar to that of the clean aerofoil case.

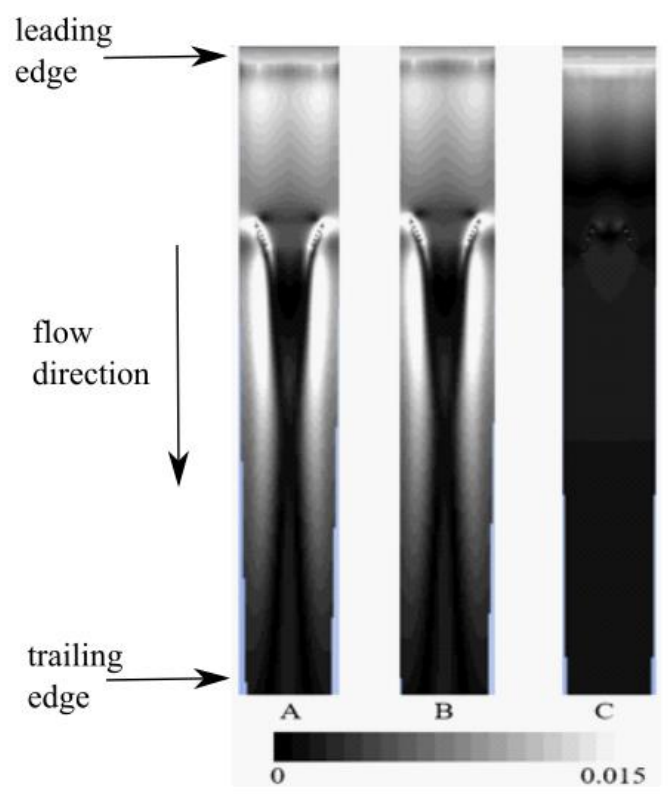

Figure22: Skin friction coefficient contours on the aerofoil surface on the suction side. AoA $=15^{\circ}$, URANS. 


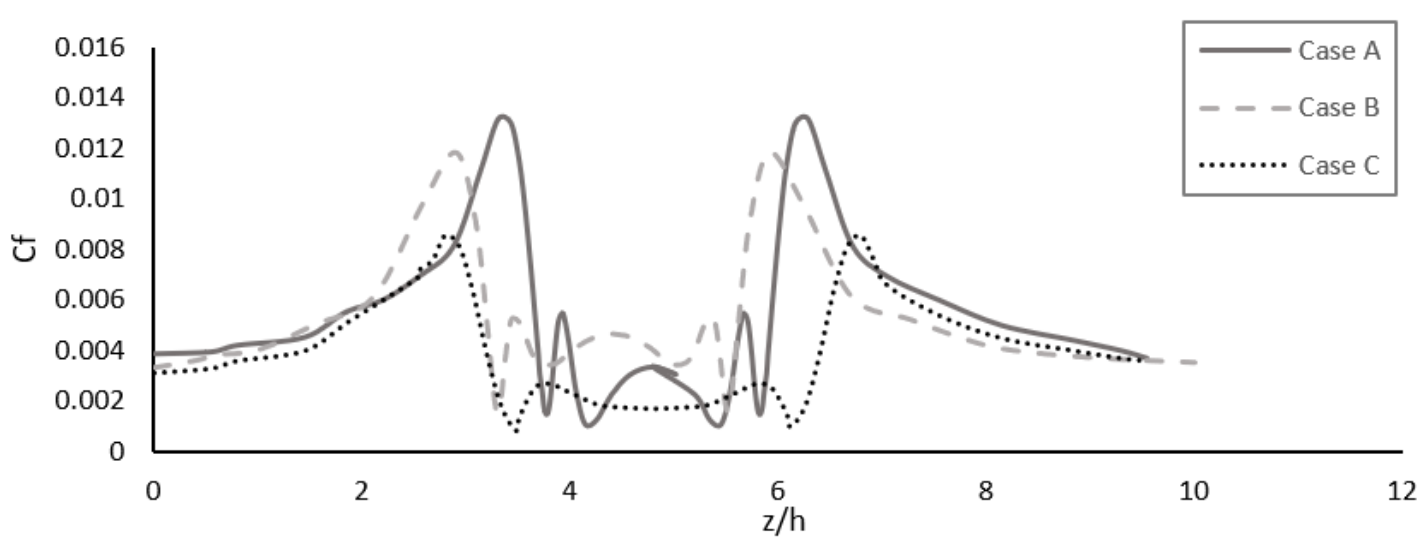

Figure 23: Skin friction selected streamwise section, $\mathrm{s} / \mathrm{h}=4, \mathrm{AoA}=15^{\circ}$, URANS.

The effectiveness of MVGs in suppressing the separation bubble is also shown in Figure 24, which compares the streamlines around the aerofoils for the cases with MVGs of different installed angles. The inflow angle of attack is again at $15^{\circ}$. In case B where the MVGs are installed at an angle of $19^{\circ}$ to the free stream, there is a relatively small vortex near the trailing edge compared to case $\mathrm{B}$, in which the MVG is installed at $22^{\circ}$. These results can also be compared to case A in Figure 20, in which the MVGs are effective in introducing the momentum from the outside to the inside of the boundary layer, and eventually suppress the flow separation. It is shown that with an increase of the installed angle from $16^{\circ}$ to $22^{\circ}$, the effectiveness of MVGs decreases. In addition, when the installed angle reaches $22^{\circ}$, the MVGs start to degrade the performance of aerofoil. A larger separation bubble is observed compared to the clean aerofoil case.

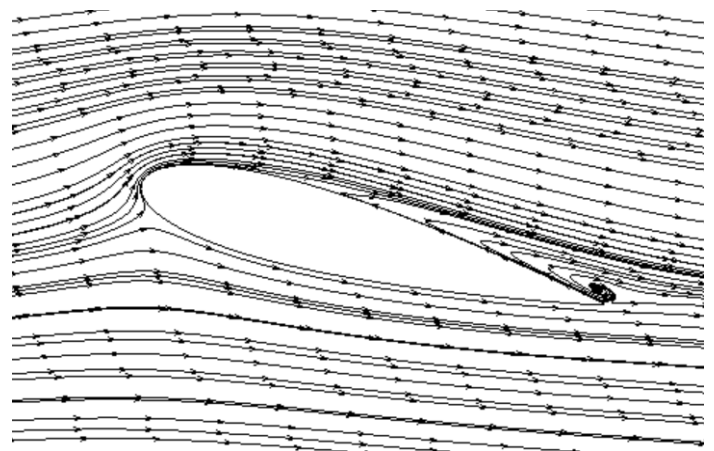

Case B

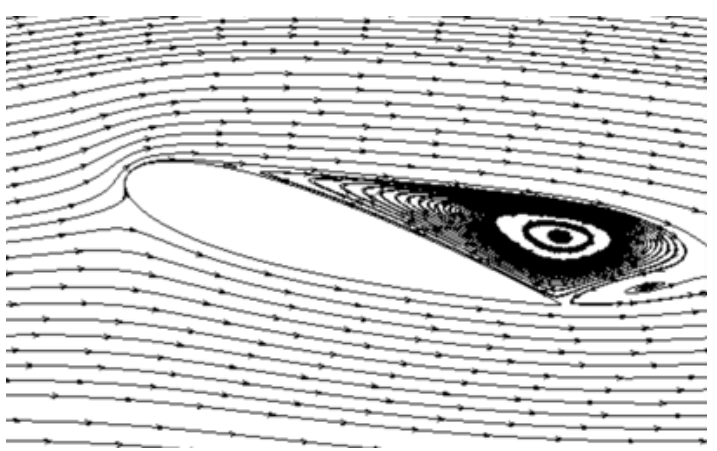

Case C

Figure 24: Comparison of streamlines around aerofoils with different MVGs at the mid-span, AoA $=15^{\circ}$, URANS

\subsubsection{MVGs of different shapes}

Apart from the location and installed angle of the MVGs, the vane can also have various shapes, such as rectangle, triangle, trapezoid and so on. Two commonly used shapes are the rectangle and the triangle as studied here. The discussion in this section is centered at the angle of attack $14^{\circ}$. Table 4 shows the effect of the shape of MVGs on the lift and drag of the aerofoil at $\alpha=15^{\circ}$. The MVGs in cases A and G have the same height and length. It was found that both MVGs improved the lift and reduced the drag compared to the clean aerofoil. The aerofoil in case A has relatively higher lift compared to case $\mathrm{G}$, while the drag is higher as well for case $\mathrm{A}$. This result in a similar lift-to-drag ratio in these two cases. 
Table 4 Comparison of drag and lift of aerofoils for different MVGs, AoA $=15^{\circ}$

\begin{tabular}{|c|c|c|c|}
\hline & $C_{L}$ & $C_{D}$ & $C_{L} / C_{D}$ \\
\hline Clean aerofoil & 0.93 & 0.084 & 11.07 \\
\hline Case A (rectangular MVGs) & 1.17 & 0.075 & 15.60 \\
\hline Case G (triangular MVGs) & 1.09 & 0.071 & 15.35 \\
\hline
\end{tabular}

The comparison between Case A and Case $\mathrm{G}$ for the vorticity magnitude is shown in Figure 25 for the downstream slices at $s / h=3$ and $s / h=7$. As indicated by the figure, the size and magnitude of the vortex generated in Case A are larger in both downstream slices, which means rectangular MVGs are more effective in vortex generation compared to the triangular ones that have smaller surface to generate the vorticity. Similar result can be found by Fouatih et al [29]. In their study, the performance of the rectangular and triangular MVGs of the same height located ar $0.3 \mathrm{c}$ with the mounting angle of $10^{\circ}$ were tested and compared on a NACA 4415 airfoil. It was found that at $\mathrm{AoA}=18^{\circ}$, the rectangular MVGs improve the lift coefficient of the base line to 1.54, while the value for the triangular MVGs was 1.48. However, the drag coefficient for the aerofoil with rectangular MVGs was slight larger than that of the aerofoil with triangular MVGs. Zhen et al. also found that rectangular VG performed better than triangular VG [16].

As the vortex convects downstream to slice $\mathrm{s} / \mathrm{h}=7$, the size of the vortex is still larger in case A. Figure 26 shows the contours of the skin friction on the suction surface of the aerofoils in cases A and G. Though the rectangular MVGs in case A and triangular MVG in case G have the same height and installed angle, the area of high skin friction behind the MVGs in case $\mathrm{G}$ is much smaller than that in case A. This indicates a weaker vortex and therefore a weaker momentum transfer between the mainstream and the boundary layer.

Case A

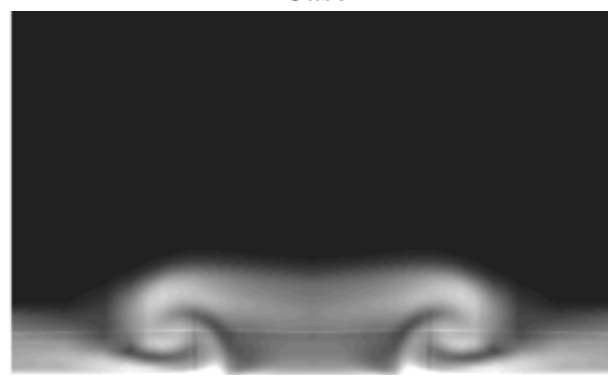

$\mathrm{s} / \mathrm{h}=3$

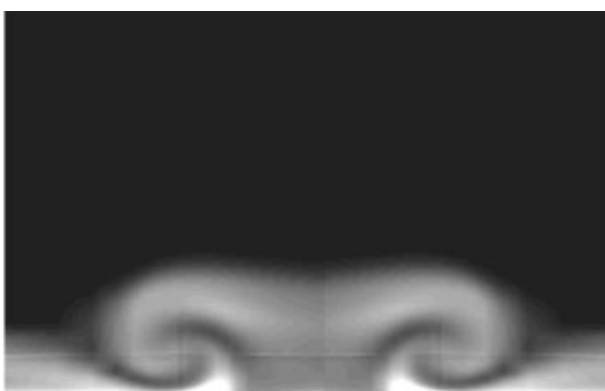

$\mathrm{s} / \mathrm{h}=7$
Case G
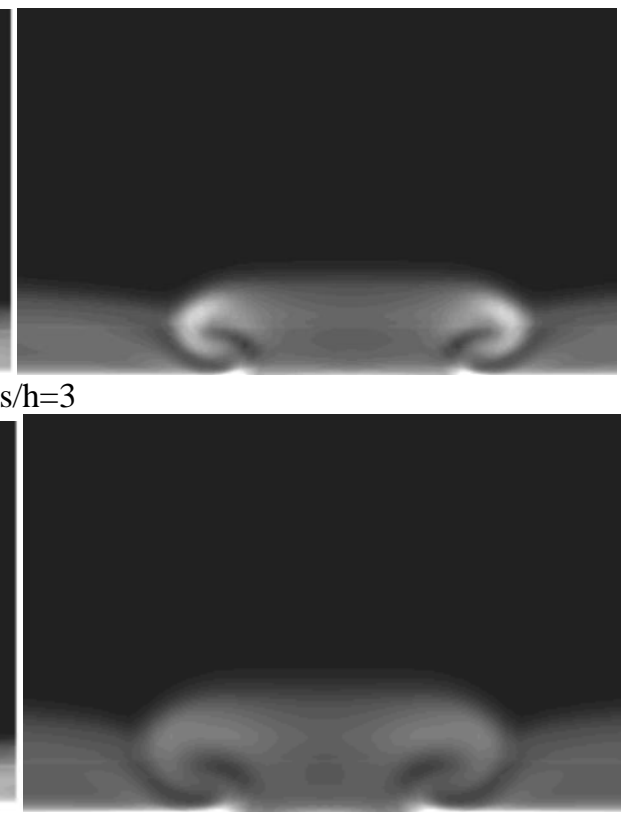

400

Figure 25: Vortcity magnitude contours at different streamwise stations downstream MVGs, $A o A=15^{\circ}$, URANS. 


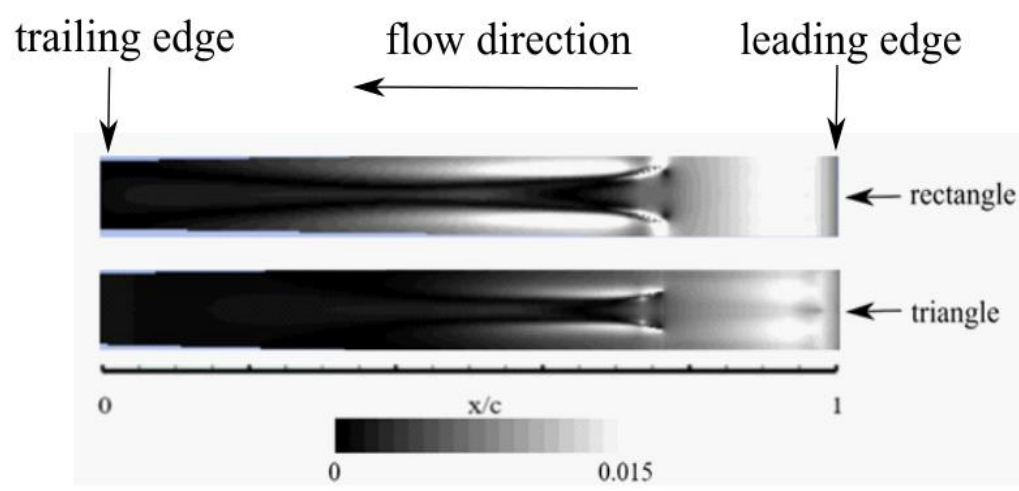

Figure 26: Skin friction coefficient contours on the aerofoil surface on the suction side, $A o A=15^{\circ}$, URANS.

The velocity contour around the aerofoils at the angle of attack of $15^{\circ}$ is revealed in Figure 27. As we can see in this figure, the boundary layer on the suction surface separates near the leading edge without MVGs installed. However, for the cases with MVGs, the separation location moves further downstream. The width of the wake is also reduced by adding MVGs and this reduction is more obvious in case A with the rectangular MVGs as compared to the triangular MVGs in case G. This is because the vortex generated by the triangular MVGs is not as strong and large as that by the rectangular ones.

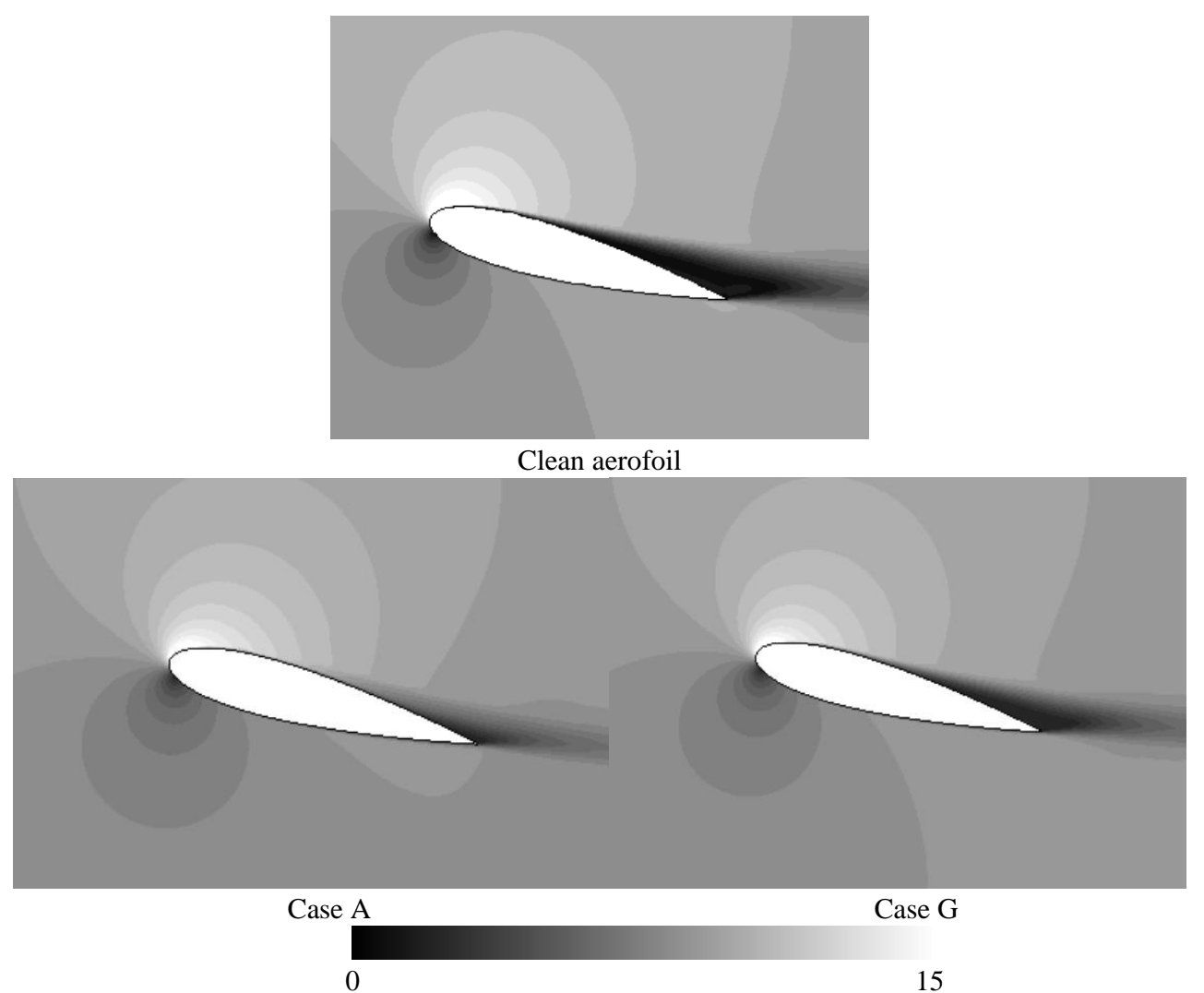

Figure 27: Comparison of velocity contours at spanwise slices midway between clean aerofoil and aerofoils with MVGs, AoA=15 ${ }^{\circ}$, URANS.

\subsubsection{The length of MVGs}


The length of the MVGs can also change the performance, and this is investigated in this section. In case $\mathrm{H}$, the MVGs' length is $\mathrm{e} / \mathrm{h}=6$, where e is the length of MVGs, which is twice as that in case A. Table 5 shows a comparison of lift and drag of the aerofoils. As can be seen in the table, at $\alpha=15^{\circ}$, the length has limited influence on the effectiveness of MVGs, as the lift and drag stay almost the same when its length is increased. When the angle of attack reaches $16^{\circ}$, although both the lift and drag in case $\mathrm{H}$ are larger than that in case $\mathrm{A}$, the increase of the drag is relatively more profound than the increase of the lift. Hence, the lift-to-drag ratio reduces with a longer MVG. This suggests that the increase in drag offsets the benefit of an increased lift for a longer MVGs.

Table 5 Comparison drag and lift for aerofoils with different MVGs

\begin{tabular}{|c|c|c|c|c|c|c|}
\hline & \multicolumn{3}{|c|}{$\mathrm{AoA}=15^{\circ}$} & \multicolumn{3}{c|}{$\mathrm{AoA}=16^{\circ}$} \\
\cline { 2 - 7 } & $C_{L}$ & $C_{D}$ & $C_{L} / C_{D}$ & $C_{L}$ & $C_{D}$ & $C_{L} / C_{D}$ \\
\hline Clean aerofoil & 0.93 & 0.084 & 11.07 & 0.80 & 0.112 & 7.14 \\
\hline Case A & 1.17 & 0.075 & 15.60 & 0.81 & 0.0813 & 9.96 \\
\hline Case H & 1.16 & 0.074 & 15.67 & 0.824 & 0.105 & 7.84 \\
\hline
\end{tabular}

\subsection{VAWT with MVGs}

\subsubsection{D mesh sensitivity analyses}

After understanding the aerodynamic performance of micro-vortex generators on an aerofoil, the effectiveness of MVGs installed on a vertical axis wind turbine is assessed in this section. The best performing MVGs studied in the previous section are selected for the wind turbine investigation. Here, large eddy simulations are performed to understand the details of the flow dynamics around the turbine blades as well as the mechanism of MVGs on improving the turbine efficiency. The length of the blade is $50 \%$ of chord length of aerofoil. To reduce the computational cost of the large eddy simulation, the tip effect is not considered. A periodic boundary condition is imposed in the spanwise direction.

The mesh sensitivity analysis has been conducted to assess the mesh quality for the LES for the flow field prediction. The 3D mesh independence study was performed only for the unmodified turbine as the base case. The power coefficient of the base case based on three grids (Mesh 4, $5 \& 6$ ) is shown in Table 6. The wall distance for all the three grids is $3.5 \times 10^{-5}$, resulting in $y+<2$. All the simulated results over estimate the power coefficient of the turbine compared to the experimental result by Balduzzi et al. [31]. Among them Mesh 4 offers the least difference with measured data. However, the discrepancy between Mesh 4 and Mesh 5 is minor, only 2.6\%. Therefore, Mesh 5 is adopted considering its reduced computational resources. The moment coefficient of one blade of the turbine is compared in Figure 28. There is no obvious difference between Mesh 4 and Mesh 5.

Table 6 Comparison of power coefficient of the VAWTs

\begin{tabular}{|c|c|c|c|}
\hline & Total cells & TSR & Power coefficeint \\
\hline Balduzzi et al. [30] & & 2.1 & 0.218 \\
\hline
\end{tabular}


599

600

601

602

603

604

605

606

607

608

609

610

611

612

613

\begin{tabular}{|c|c|c|c|}
\hline Mesh 4 & $4.92 \times 10^{7}$ & 2.1 & 0.228 \\
\hline Mesh 5 & $3.59 \times 10^{7}$ & 2.1 & 0.234 \\
\hline Mesh 6 & $1.76 \times 10^{7}$ & 2.1 & 0.263 \\
\hline
\end{tabular}

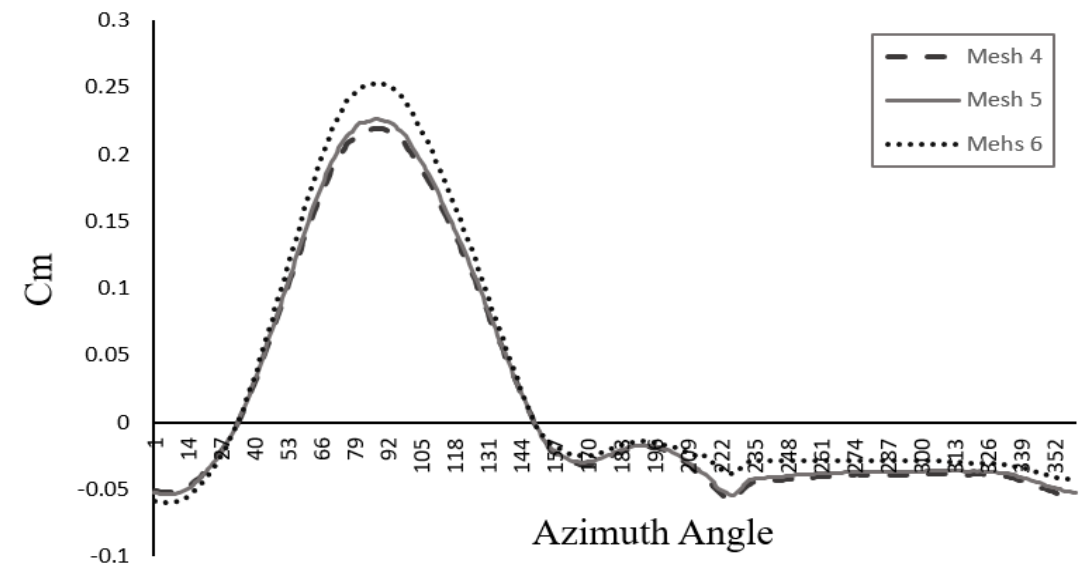

Figure 28: Moment coefficient for different meshes.

The LES results of an H-type 3 blade turbine without MVGs are compared to the results available in the literature. Figure 29 shows the comparison of the measured data and the CFD results in terms of power coefficient versus tip speed ratio. The rotors in the current study are the same as in the experiment and CFD in [30].

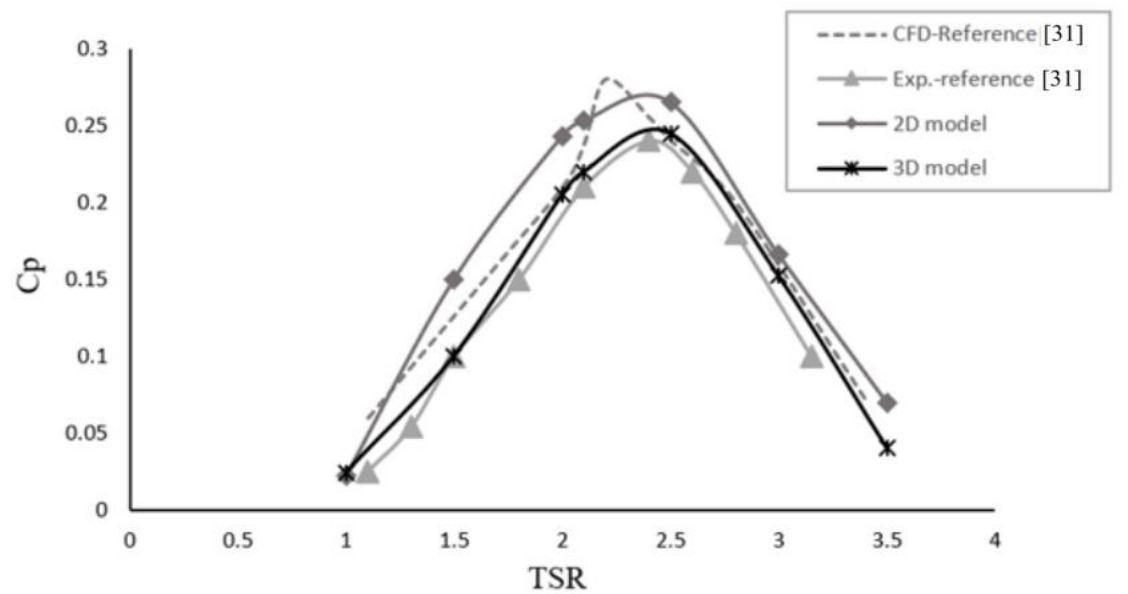

Figure 29: Power coefficient comparison between experiment and CFD results, LES [30].

In order to setup the time step for three-dimensional simulation and assess how it affects the results, a time-step sensitivity analysis was performed. Three different values of time step were chosen for testing. They are $\Delta \mathrm{t}=1 \mathrm{e}-4 \mathrm{~s}, 3 \mathrm{e}-4 \mathrm{~s}, 6.7 \mathrm{e}-4 \mathrm{~s}$, where one time period of the turbine rotation is $0.33 \mathrm{~s}$ at $\mathrm{TSR}=2$. The moment coefficient with different time steps was investigated as in Figure 30. It was found that the result of $\Delta \mathrm{t}=6.7 \mathrm{e}-4 \mathrm{~s}$ agrees well with a smaller time step, thus the time step step size of $6.7 \mathrm{e}-4 \mathrm{~s}$ is used to keep the computational cost to a feasible level. 


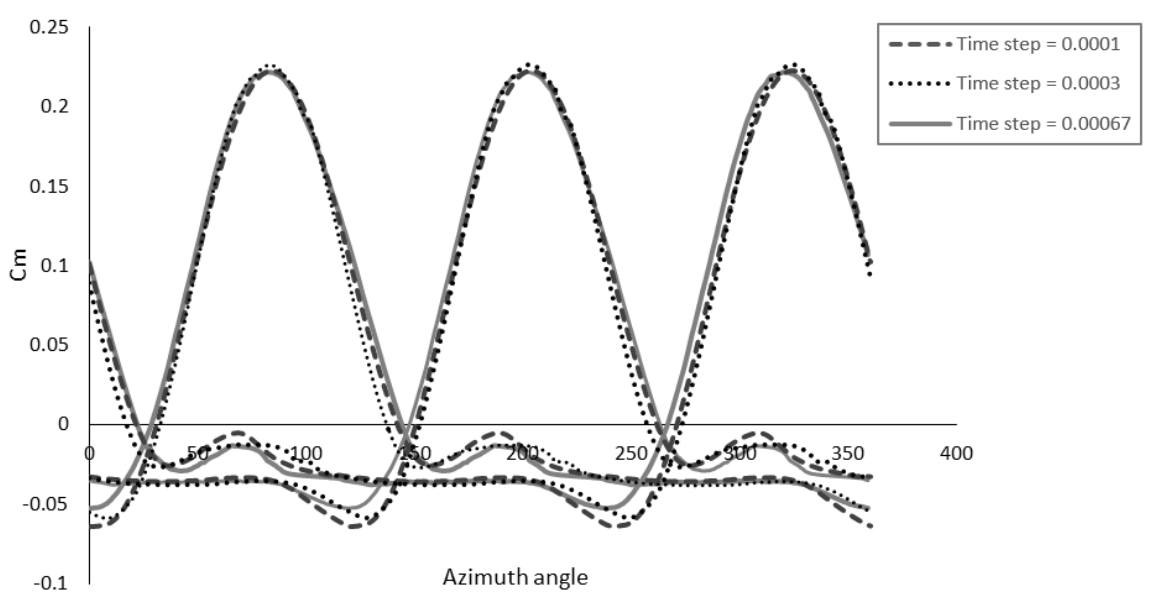

Figure 30: Moment coefficient of the three blades for different time step sizes, TSR=2.5, LES.

For the lift-based turbines, the angle of attack variation for one cycle should be investigated as it has a great influence on the lift generation. Figure 31 depicts the variation of angle of attack at different rotor blade azimuth angles and for different tip speed ratios over a full cycle. The maximum angle of attack decreases as the TSR increases. At low TSRs, VAWTs encounter a wide range of angles of attack as shown in Figure 30. As the static stall angle of aerofoil NACA 0018 at $R e_{c}=1.6 \times 10^{5}$ is $14^{\circ}$. It is clearly found that for the lower TSR, the turbine blades experience a larger part of azimuth angles that exceeds the static stall angle in one revolution. At TSR $=1.5$, during most of the revolution the blade is in deep stall condition.

Figure 32 shows the lift and drag variations for a wide range of angles of attack (AoA) from $0^{\circ}$ to $40^{\circ}$. This range covers the AoA that turbine blades encounter in one revolution at $\mathrm{Re}=1.6 \times 10^{5}$. The effect of MVGs for the aerofoil around the stall angle has been already discussed in detail. The lift drops significantly after the stall angle and then slightly increases with the increasing of AoA. It is clear that at AoA from $28^{\circ}$ to $40^{\circ}$, the lift of a clean aerofoil is slightly higher that the aerofoil with MVGs. On the other hand, the MVGs have no visible influence on the drag of the aerofoil as shown in Figure 32(right) for those high AoAs. This can be explained by the fact that the MVGs are inside the massive flow separation region of the stall and cannot function as intended, i.e. inject fresh air from the outer boundary layer to the inner one.

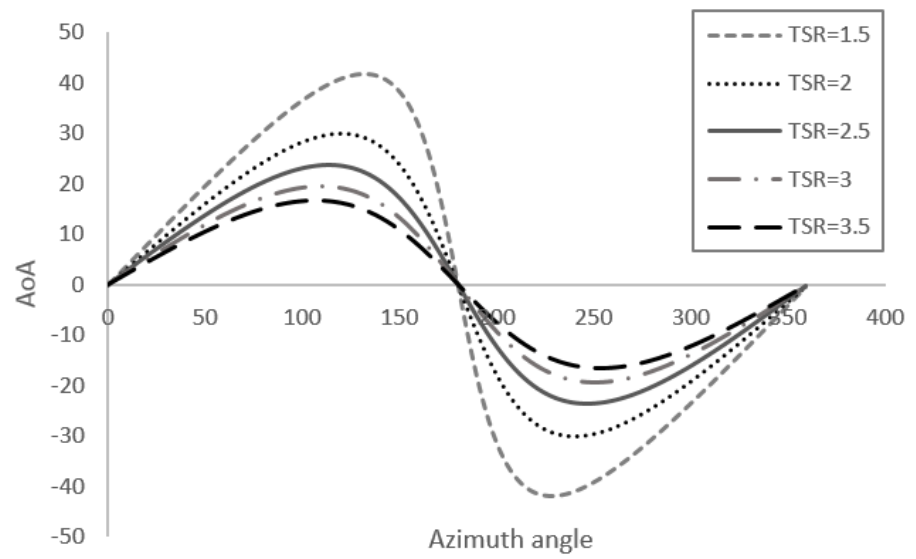

Figure 31: Angle of attack (AoA) variation in one reolution at various TSRs. 


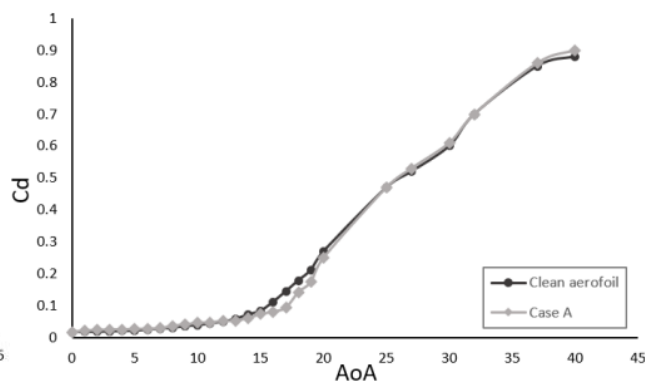

Figure 32: Lift (right) and drag (left) comparison for clean aerofoil and aerofoil with MVGs A, URANS.

\subsubsection{Effect of location for the performance of MVGs}

The function of MVGs A and $\mathrm{E}$ on the turbines is investigated in this section as these two configurations of MVGs were found to be the most effective in improving the aerodynamic performance of a single aerofoil. The torque and the power curves of the clean VAWT and VAWTs with rectangle MVGs of the two locations at the wind speed of $8 \mathrm{~m} / \mathrm{s}$ are presented in Figure 33. All the performance curves start at a lower value and peaking before dropping to a lower value again. The results were computed with LES. As we can see in this figure, the effect of the MVGs on the performance of a VAWT varies with the Tip Speed Ratio (TSR). At low TSRs, from 1 to 2, the VAWT with and without MVGs have a similar performance. This is because at low TSRs, the turbine blades are considerably at post stall condition during most of the part of the turbine rotation cycle as discussed in the last section. As the MVGs have nearly no effect at angles of attack much higher than the stall angle, their effect was limited on the performance of turbines at low TSRs. When the TSR is larger than 2, adding MVGs with a suitable configuration gives improvement of performance. Compared to other cases, the MVGs located at $20 \%$ chord length of the blade's profile give the best performance at TSR $=2.5 \& 3$. This is consistent with the observation made for the single aerofoil.
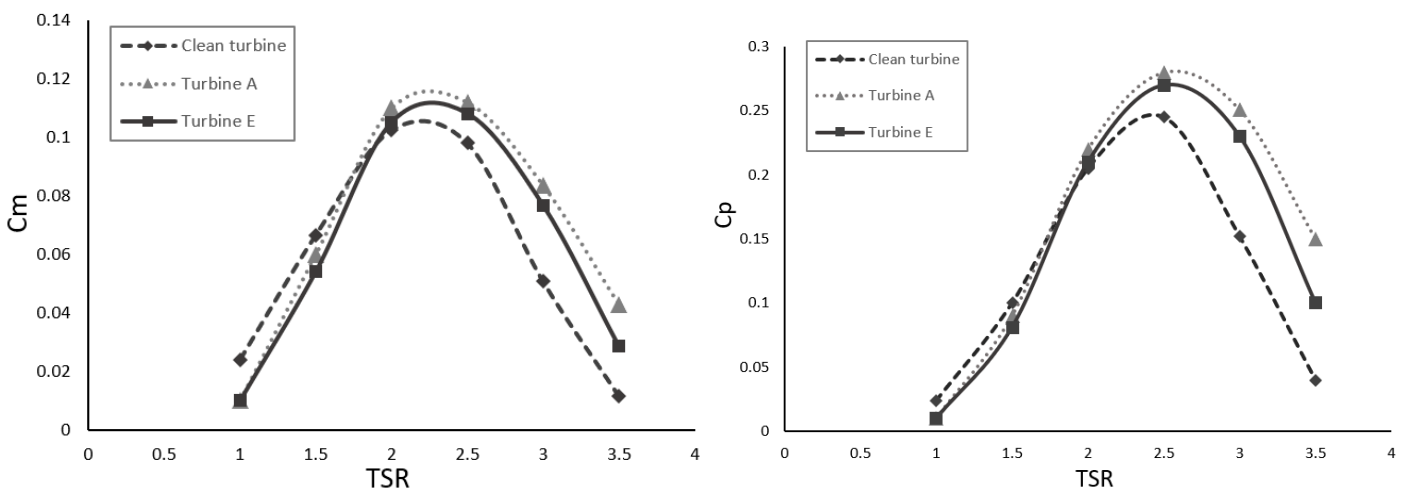

Figure 33: Variations of the moment coefficient and power coefficient as functions of azimuth angle for one blade of various MVGs, LES.

In order to understand the mechanism of the efficiency improvement due to MVGs, the phase-averaged moment coefficient of one blade for one rotation cycle VAWTs is presented in Figure 34. It is evident that most of the wind energy is captured in the first half cycle. For the second half cycle, the moment coefficient $\mathrm{Cm}$ of all turbines is low due to the fact that the blade is traveling within the wake of the upstream blade. The 
main differences of $\mathrm{Cm}$ are at the first half cycle where the azimuth angle $\theta$ ranges between $75^{\circ}$ and $160^{\circ}$, and hence the flow separation appears because of the relatively large AoA the blades encounter as shown in Figure 31.

At low azimuth angles from $0^{\circ}$ to $80^{\circ}$, the $C m$ of all cases follows a similar trend: the moment coefficient increases as the azimuth angle rises. This is because the lift increases with the AoA before stall occurs. When the azimuth angle increases to $80^{\circ}$, where $\mathrm{AoA}=14^{\circ}$ at $\mathrm{TSR}=2.5$, the rotor blades start to stall and the moment coefficient begins to decline from its peak value. The maximum $C_{m}$ and the azimuth locations of the peak value vary in different cases

As shown in Figure 34 (left), when the azimuth angle increases to around $80^{\circ}$, the moment coefficient of the clean turbine reaches its peak value of 0.237 and starts to decline. However, for other cases, the moment coefficient continues to rise. With the increase of azimuth angle, $\mathrm{Cm}$ of turbine $\mathrm{A}$ is the last one to reach its peak value as compared to other cases, for both TSRs of Figure 34. In addition, a maximum value of $\mathrm{Cm}$ is observed in turbine A. Compared to the clean turbine, we can conclude that MVGs can improve the performance of VAWTs, and the results are consistent to that of an isolated aerofoil discussed in the last section. A similar result at TSR $=3$ is shown in Figure 34 (right), turbines A and E produce more power output at the first half of the cycle after stall as compared to the clean turbine.

On the second half of the cycle, the angle of attack is negative as shown in Fig.31, which leads to the MVGs being the pressure side of the aerofoil instead of the suction side. Hence, the MVGs have no effect on the flow separation and no noticeable difference between the clean turbine and the turbines equipped with MVGs is observed.
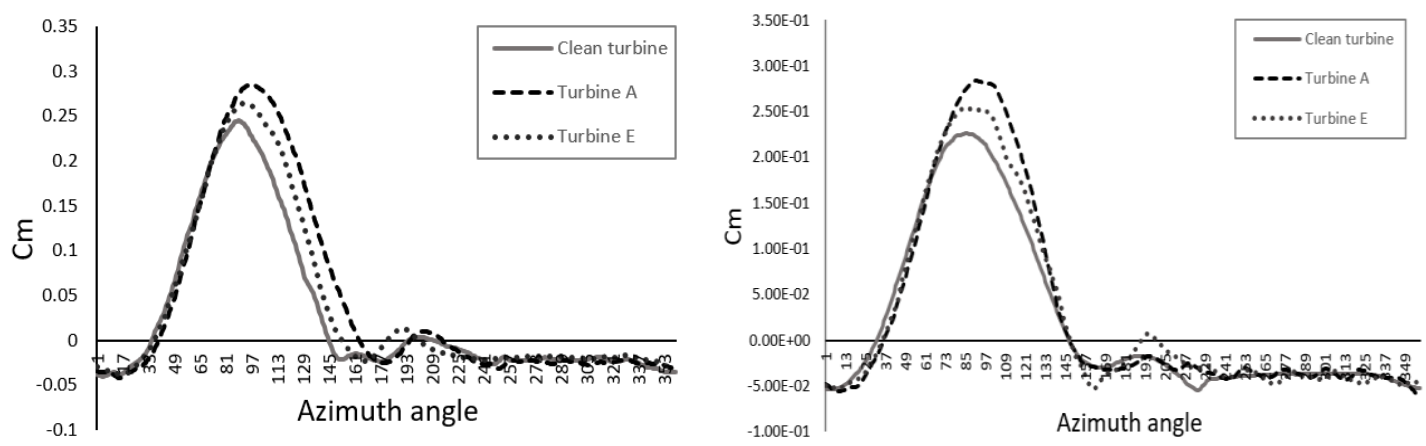

Figure 34: Blade phase-averaged moment coefficient comparison of various turbines TSR=2.5 (left) $\mathrm{TSR}=3$ (right), LES.

The overall moment, which combines all the three blades is another parameter that can be used to evaluate the turbine performance. Figure 35 plots the variation of the overall phase-averaged moment coefficient of various turbines over a full operational cycle at $\mathrm{TSR}=3$. All the cases show a similar trend and turbine A offers the maximum value of moment coefficient, which is consistent with the previous analysis. 


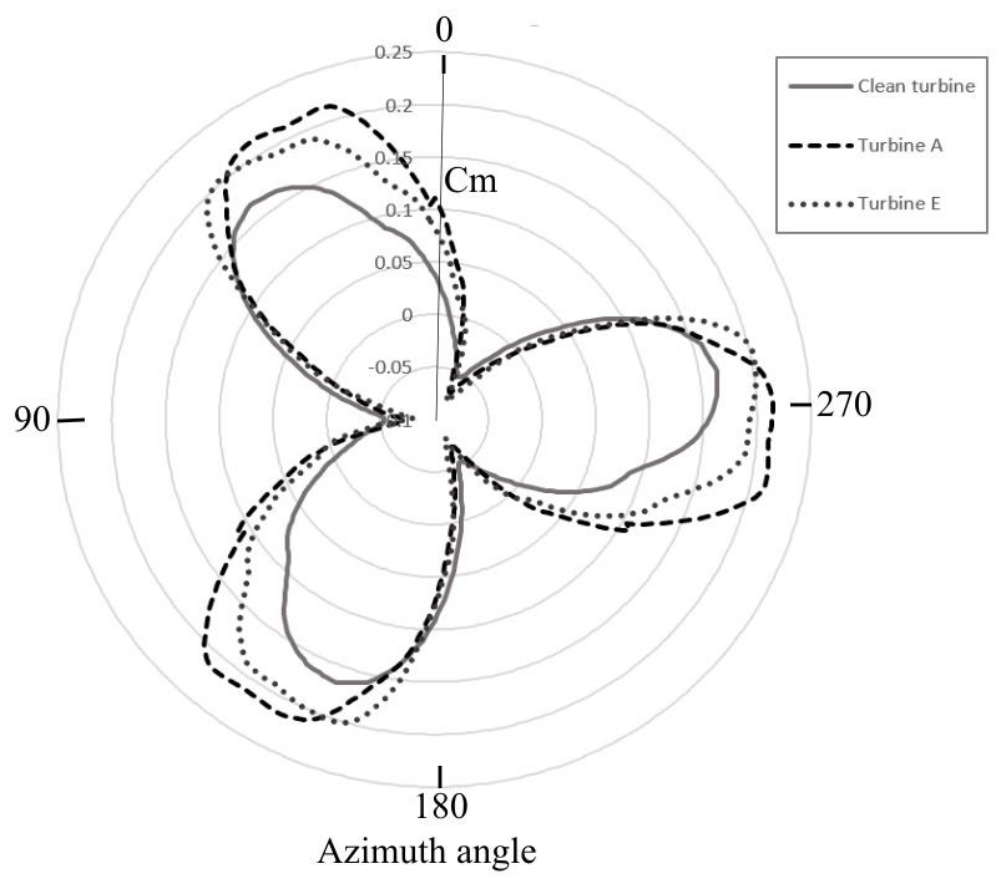

Figure 35: Moment coefficient variation with azimuth angle clean turbine and turbine A, B\&C, TSR=3, LES.

Figure 36 shows the contour of the vorticity magnitude around the blade profile of different turbines at $\mathrm{TSR}=2.5$. The $\mathrm{MVG}$ s offer a dramatic change in the pressure distribution on the suction side of the aerofoil. At an azimuth angle of $\theta=120^{\circ}$, the profile exceeds the stall angle and mild separation starts to occur in the boundary layer of the clean turbine. Two spanwise vorticity rolls can be observed: one originated from the leading-edge separation and the other separation occurs near the trailing edge. The separation point in turbine $\mathrm{A}$ is farther away from the leading edge of the aerofoil compared to the clean turbine case and is consistent to a higher lift and torque generation. In turbine $\mathrm{E}$, the flow separation on the suction side of blade is weaker compared to the clean turbine as well.

Figure 37 shows a similar result at $\mathrm{TSR}=3$. When the turbine blade rotates to $\theta=130^{\circ}$, the flow separation of the clean turbine is more profound as compared to the turbines with MVGs A and E again pointing to the benefits of the MVGs on delaying flow separation.

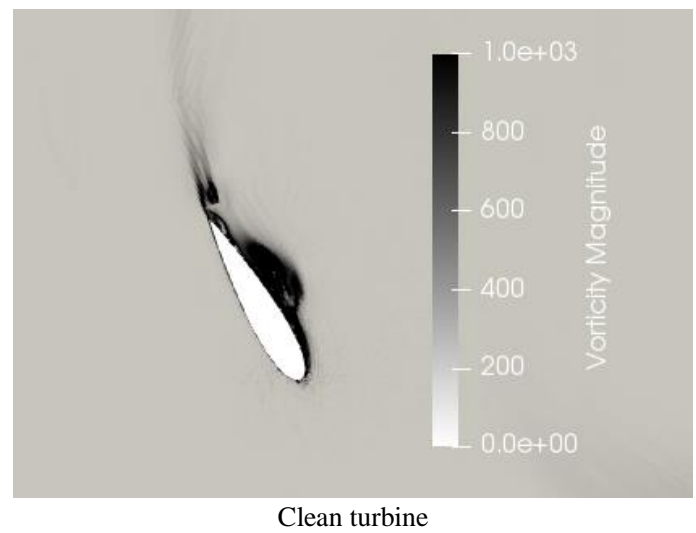




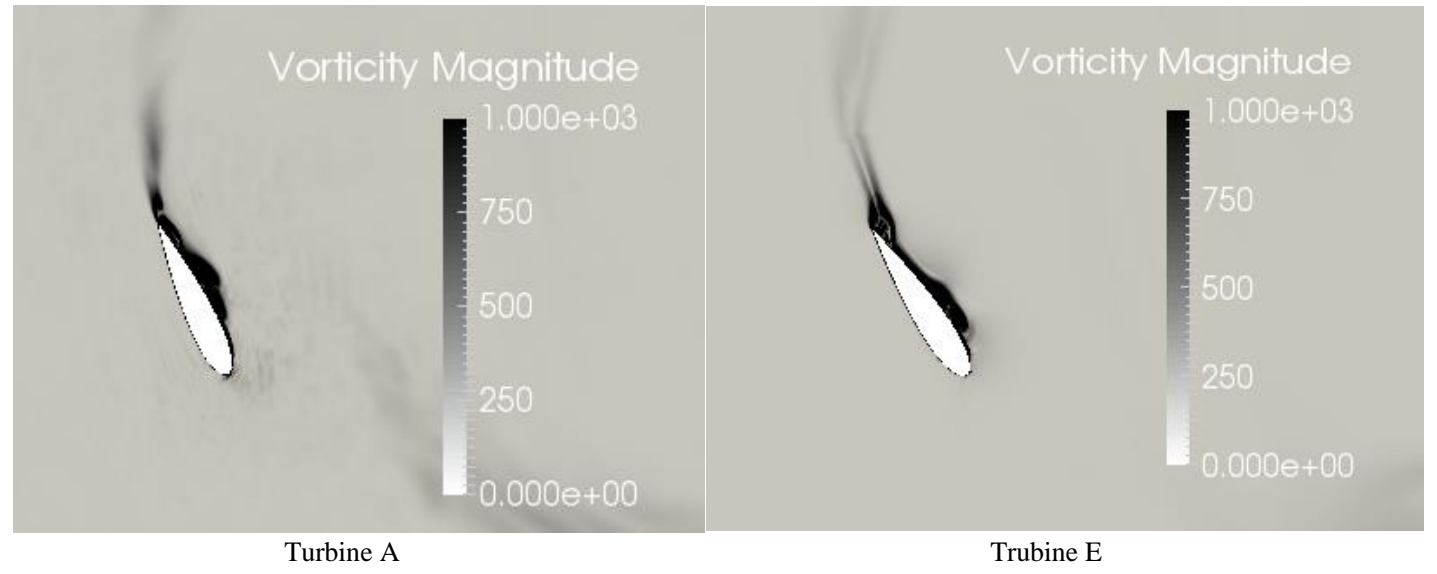

Figure 36: Vorticity magnitude comparison of various turbines at $\theta=120^{\circ}$, TSR=2.5, LES.

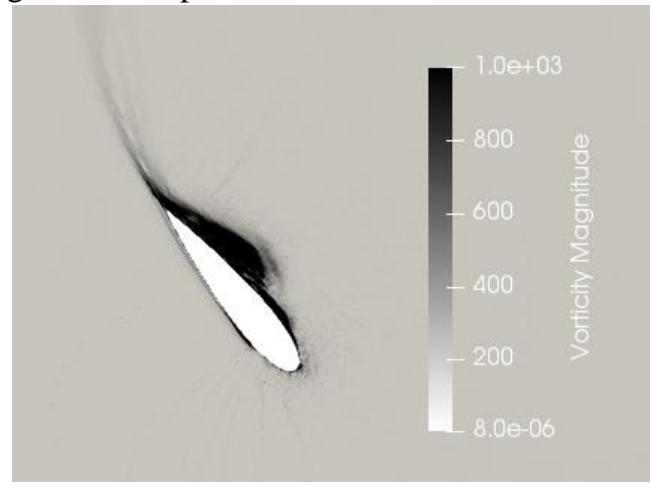

Clean turbine

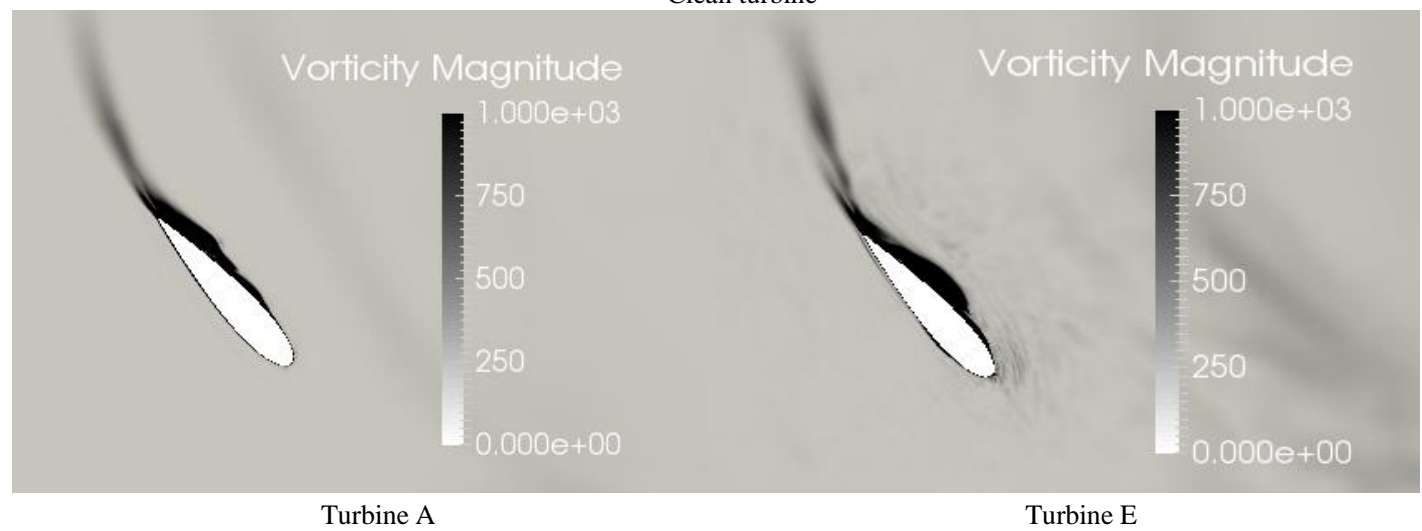

Figure 37: Vorticity magnitude comparison of various turbines at $\theta=130^{\circ}$, TSR $=3$, LES.

The static pressure field is shown in Figure 38 for a blade aerofoil section at $\theta=90^{\circ}$. As we can see in this figure, this qualitative comparison shows some significant differences in the pressure distribution of the various turbines. The area of the region with a low pressure on the suction side of turbine blade is larger for turbine A and turbine $\mathrm{E}$ than the clean turbine. This corresponds to a larger pressure difference, leading to a larger moment generation at this azimuth angle for turbines $\mathrm{A}$ and $\mathrm{E}$. The result agrees well with the moment coefficient distribution as Figure 34. The power output of turbine D is the lowest at $\theta=90^{\circ}$ as compared to the other turbines. 


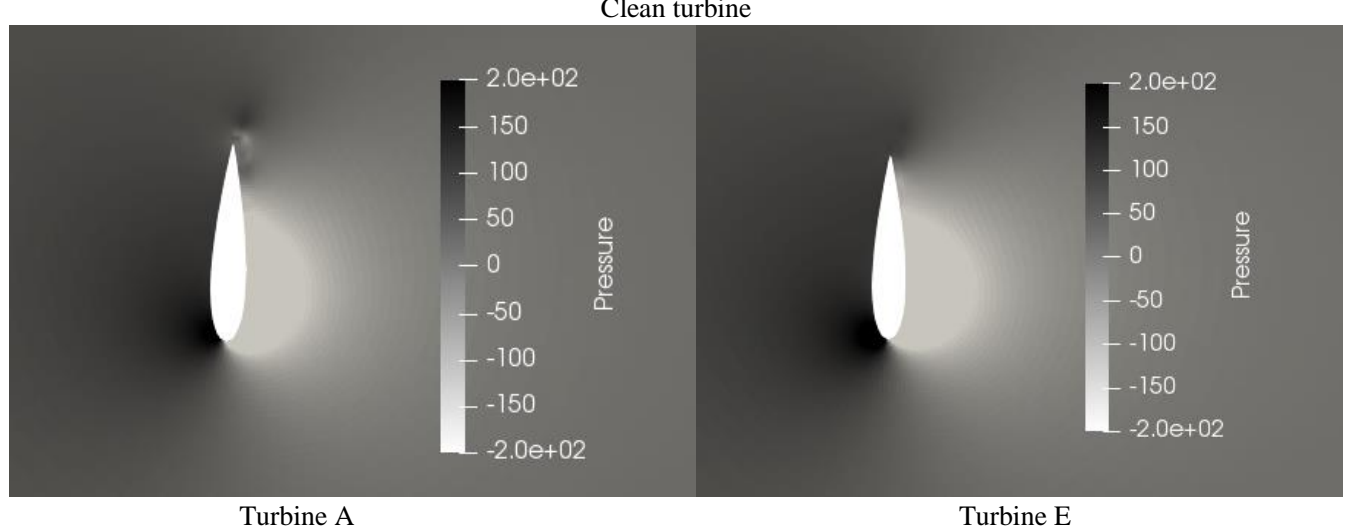

Figure 38: Pressure contour of various turbine blades at $\theta=90^{\circ}$, $\mathrm{TSR}=2.5$, LES.

738

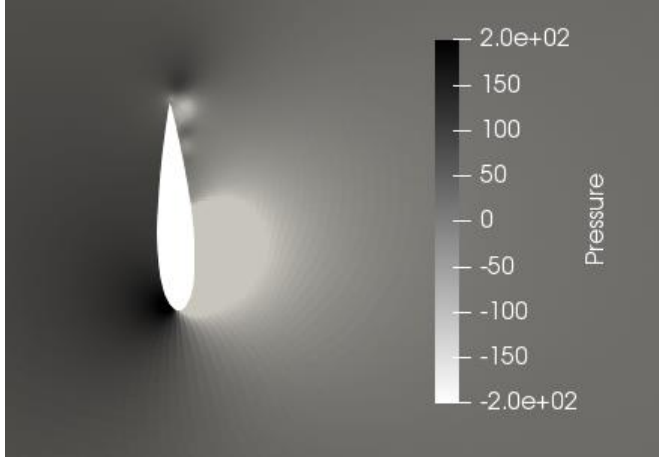

Clean turbine

\subsubsection{Effect of installed angle on the performance of MVGs}

Figure 39 shows a comparison between the clean turbine and the turbine with MVGs of various installed angles in terms of moment coefficient and power coefficient versus TSR. It is noticeable that the installed angle can affect the aerodynamic performance of the VAWTs. At low TSR from 1 to 2, the three turbines provide similar performance. When the TSR increases to 2.5, the power coefficients of turbine A and B follow each other very closely and produce more power output compared to the clean turbine. MVG $\mathrm{C}$ slightly degrades the power output of the turbine at the medium range of tip speed ratios of 2 to 3 .
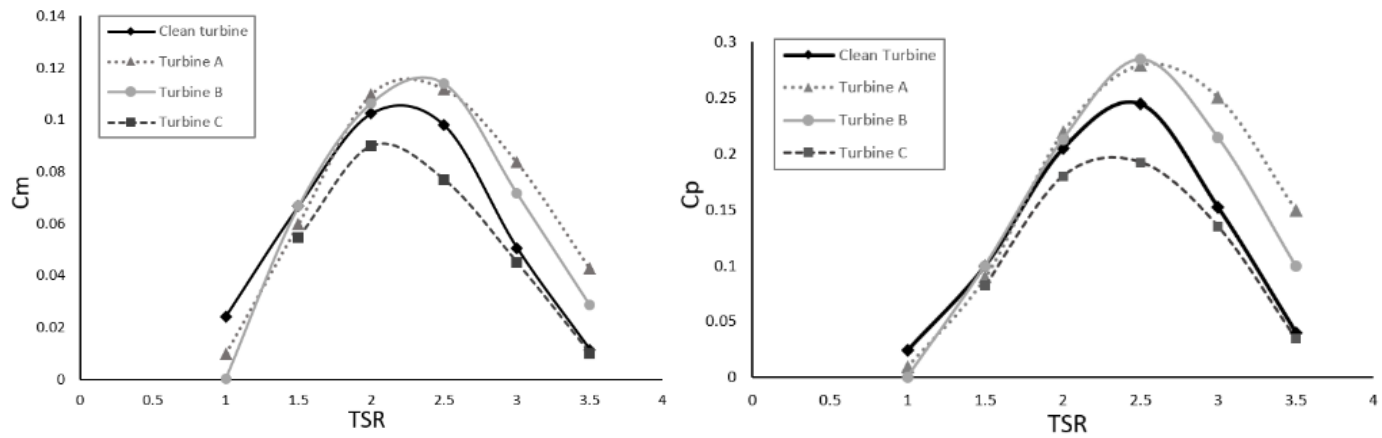

Figure 39: Moment coefficient (right) and power coefficient (left) comparison of different turbines, LES.

The comparison between these four models in terms of the instantaneous moment coefficient of a single blade operating at TSR $=2.5 \& 3$ for one revolution is presented in Figure 40. At both TSRs, the torques generated from these four turbines are found to increase with a very similar trend from $\theta=0^{\circ}$ to $80^{\circ}$, which is similar to the models 
discussed before. A discrepancy starts to occur in the clean turbine and turbine $\mathrm{C}$, which reach the peak value earlier as compared to the other two models. The moment coefficient for turbine A and turbine B continues to increase before reaching the peak value at around $\theta=95^{\circ}$. In the azimuth angle ranging from $80^{\circ}$ to $150^{\circ}$, turbines $A$ and $B$ show a significant improvement in power output. At $\mathrm{TSR}=2.5$, turbine $\mathrm{B}$ achieves the highest peak value of moment coefficient and at TSR=3, turbine A performs better as compared to the other models. All models generate a mild negative torque in the second half revolution and there is no significant difference between them at TSR $=3$.
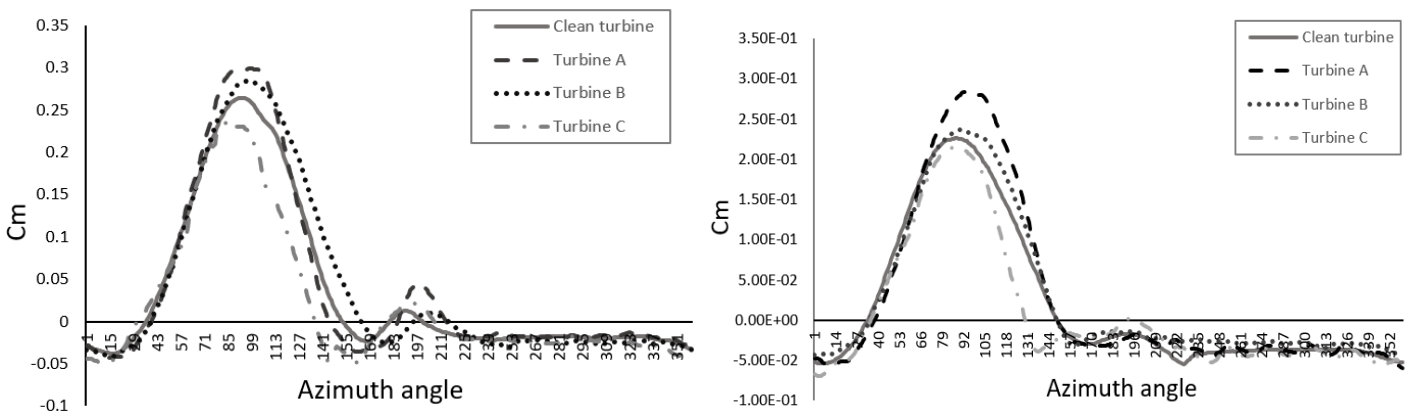

Figure 40: Blade phase-averaged moment coefficient comparison of clean turbine and turbine A, B \& C, TSR=2.5(left) TSR=3(right), LES.

When the blades are at the azimuth angle of $120^{\circ}$, the flow becomes highly separated due to the high angle of attack, showing a dynamic stall at this stage, which is related to a sharp torque decrease shown in Figure 39.

Figure 41 shows the distribution of the vorticity at the azimuth angle $135^{\circ}$. From the visualization of the vorticities, the flow separation is stronger in the clean turbine as compared to turbines A and B demonstrating the effectiveness of the MVGs.
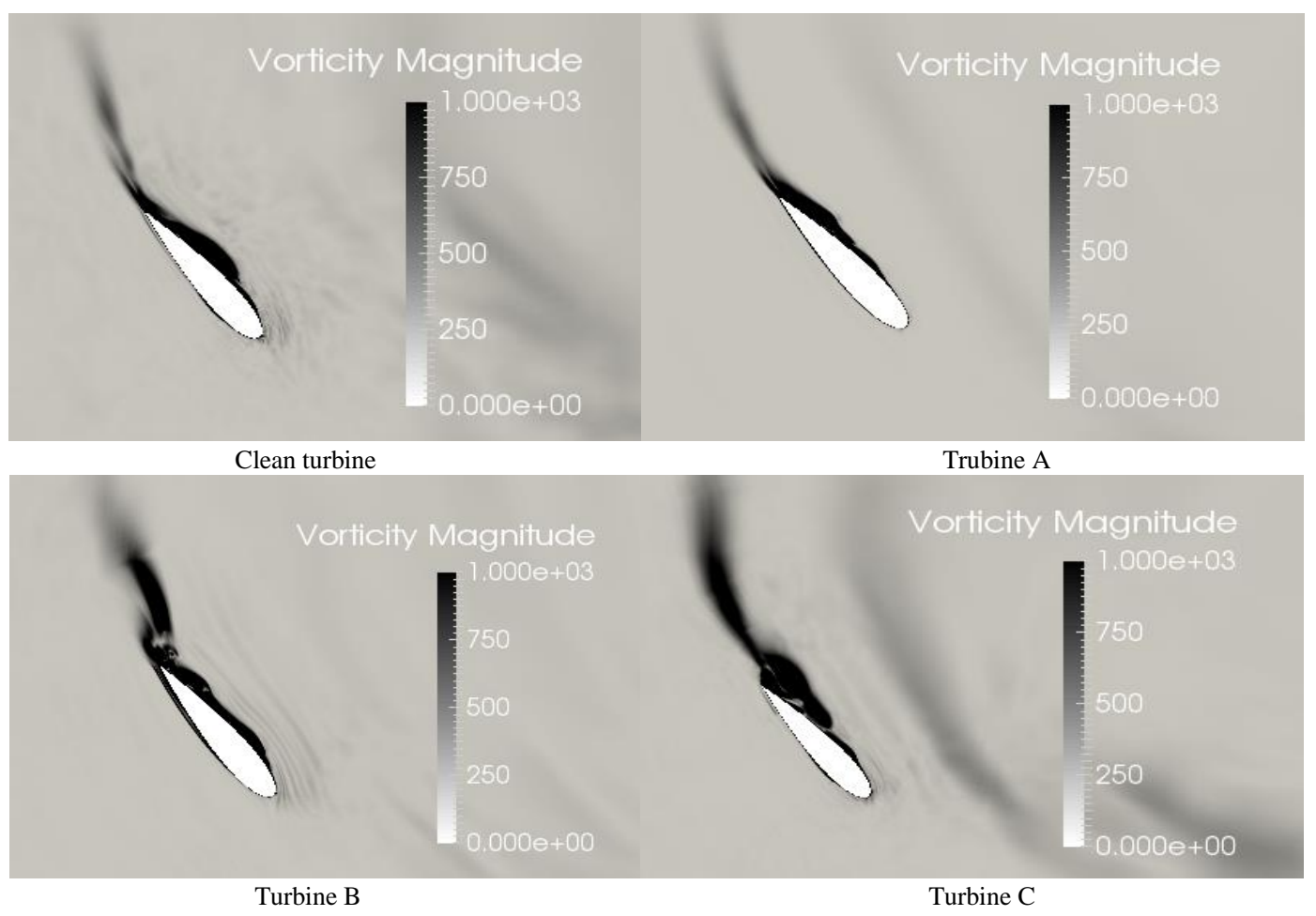

Figure 41: Mid span vorticity magnitude comparison of various turbines at $\theta=130^{\circ}$, TSR $=3$, LES. 
The static pressure as relative to the atmosphere pressure contour is shown in Figure 42. All blades show similar pattern of largest pressure difference between the pressure (left) and suction (right) side near the tip as expected from aerofoil aerodynamics. The effect of the MVGs is clear on the suction side where it is mounted than on the pressure side. From turbines A and B, we can see that the low pressure region goes further into the trailing edge than in the clean turbine contributing to high pressure difference and thus higher lift. However, turbine $\mathrm{C}$ blade shows a reduced pressure near the trailing edge due to the vortex shedding and thus reduced lift as compared to turbines A and B. Its reduced pressure region near the leading edge. All this contributed to the lower $C_{m}$ by turbine $\mathrm{C}$ at $\theta=90^{\circ}$ seen in the Figure 39 (left).
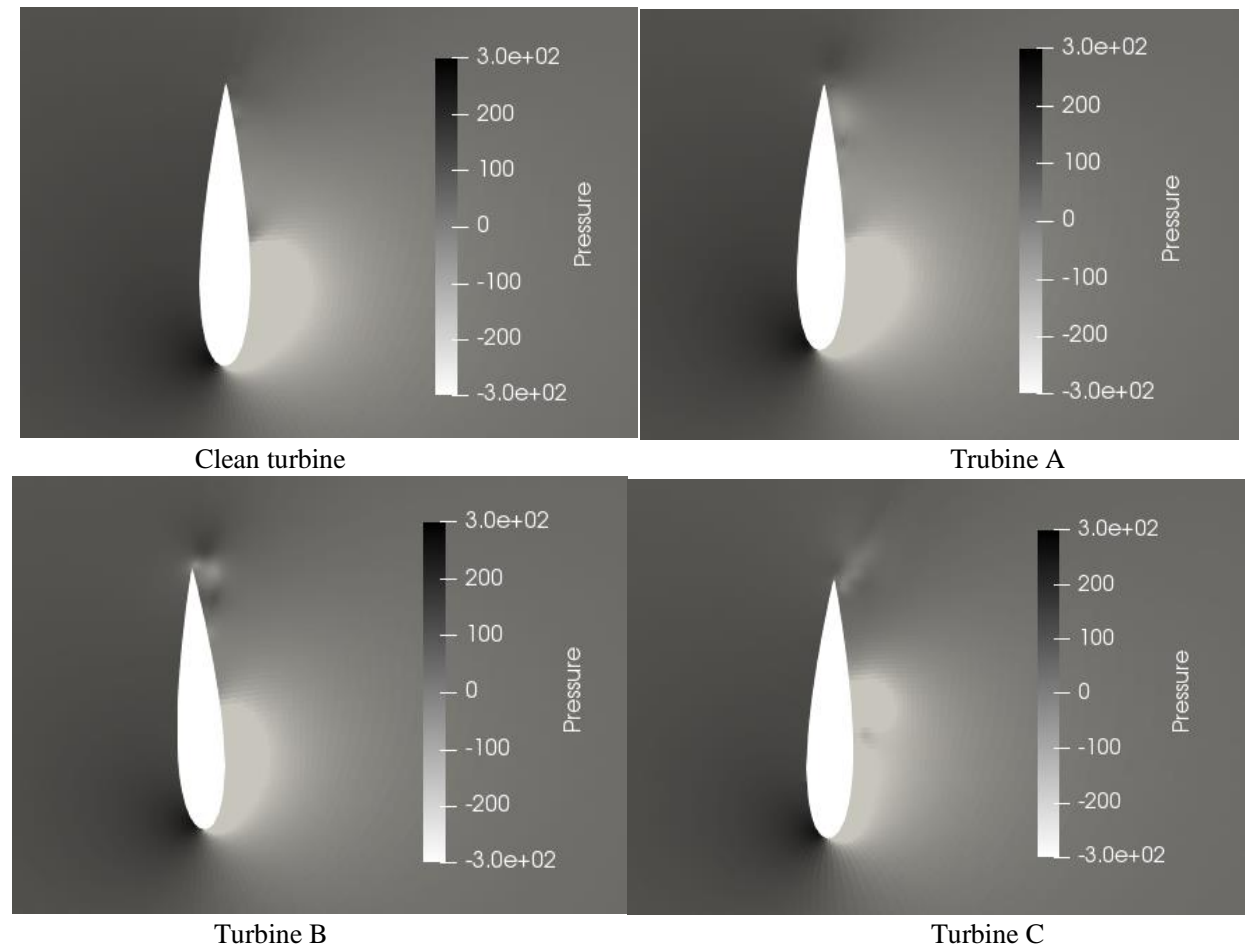

Figure 42: Mid span pressure contour of various turbine blades at $\theta=90^{\circ}$, TSR $=2.5$, LES.

\section{CONCLUSIONS}

The purpose of the present study was to determine an effective passive flow control technique to enhance the aerodynamic performance of the NACA 0018 aerofoil commonly used in the wind industry and an associated $\mathrm{H}$-type vertical axis wind turbine (VAWT). Firstly, the dynamics of an MVG vane embedded in the boundary layer of a flat plate was investigated. The time-averaged flow field is found to compare well with the published experimental results.

Several MVGs of various configurations implemented on the suction side of the aerofoil and turbine blades are numerically investigated. The results show that MVGs have a significant effect on both the aerofoil and the turbine as a whole. With the MVGs of a suitable design, both the lift coefficient and lift-to-drag ratio can be increased at high angles of attack and the stall angle delayed. The turbine blades with MVGs show a better capability of power generation in comparison to clean blades, having a potential impact on future VAWT design.

The following conclusions can be highlighted: 
1. For the isolated aerofoil NACA 0018, the optimum positioning of the MVGs was found to be at $20 \%$ chord length along the suction side of the aerofoil with a rectangular shape and installed angle of $16^{\circ}$. The stall angle delays to $16^{\circ}$ from $14^{\circ}$ with the installation of MVGs. The maximum lift is improved by $37.5 \%$ from 0.96 to 1.32 , while the drag decrease from to 0.178 to 0.137 at post stall condition $\alpha=18^{\circ}$.

2. For the VAWT, a similar conclusion was obtained. The best performance was found for turbine $A$ at high TSRs from $\lambda=2.5$ to 3.5 in comparison with the other models. Among various TSRs, the MVG A has the most significant effect at $\mathrm{TSR}=3$, where the power coefficient increases by more than $50 \%$ to 0.24 . This investigation illustrates that MVGs can be an effective technique for delaying flow separation control in operating VAWTs at high TSRs.

\section{ACKNOWLEDGMENT}

The first author thanks CSC China and QMUL for supporting her PhD studentship. This work was supported by ARCHER via UK EPSRC Turbulence Consortium grant EP/R2932611, China Scholarship Council and the Zhejiang University/ University of Illinois at Urbana-Champaign Institute. It was led by Principal Supervisor Eldad Avital and Jiahuan Cui.

\section{REFERENCE}

1. KC, A., Whale, J., \& Urmee, T. (2019). Urban wind conditions and small wind turbines in the built environment: A review. Renewable Energy, 131, 268-283.

2. Shen, X., Avital, E., Paul, G., Rezaienia, M. A., Wen, P., \& Korakianitis, T. Experimental study of surface curvature effects on aerodynamic performance of a low Reynolds number airfoil for use in small wind turbines. Journal of Renewable and Sustainable Energy, 8(5), 053303, 2016.

3. M. M. A. Bhutta, N. Hayat, A. U. Farooq, Z. Ali, S. R. Jamil, and Z. Hussain. Vertical axis wind turbine-a review of various configurations and design techniques. Renewable and Sustainable Energy Reviews, 16(4):1926-1939, 2012.

4. Battisti, L., Benini, E., Brighenti, A., Dell'Anna, S., \& Raciti Castelli, M. (2018). Small wind turbine effectiveness in the urban environment. Renewable Energy, 129, 102-113.

5. HD Taylor, "The elimination of diffuser separation by vortex generators," Technical Report No. R-4012-3, United Aircraft Corporation, 1947.

6. Calarese W, Crisler WP, Gustsfson GL. Afterbody drag reduction by vortexgenerators. AIAA Paper 85-0354, AIAA 23rd Aerospace Sciences Meeting, Reno, NV, January 14-17, 1985.1

7. Lin, J. C., Robinson, S. K., McGhee, R. J., \& Valarezo, W. O. (1994). Separation control on high-lift airfoils via micro-vortex generators. Journal of Aircraft, 31(6), 1317-1323.

8. J. Johansen, N. N. Sørensen, M. Reck, M. Hansen, A. Stuermer, J. Ramboer, C. Hirsch, J. Ekaterinaris, S. Voutsinas, and Y. Perivolaris. Know-blade task-3.3 report: Rotor blade computations with 3d vortex generators, 2005.

9. L. Gao, H. Zhang, Y. Liu, and S. Han. Effects of vortex generators on a blunt trailing-edge airfoil for wind turbines. Renewable Energy, 76:303-311, 2015. 
10. R. J. Volino. Separation control on low-pressure turbine airfoils using synthetic vortex generator jets. In ASME Turbo Expo 2003, collocated with the 2003 International Joint Power Generation Conference, pp. 845-859. American Society of Mechanical Engineers, 2003.

11. R. G. Hibbs, S. Acharya, Y. Chen, D. E. Nikitopoulos, and T. A. Myrum. Heat transfer in a twopass internally ribbed turbine blade coolant channel with cylindrical vortex generators. In ASME 1996 International Gas Turbine and Aeroengine Congress and Exhibition, pp. V004T09A051-V004T09A051. American Society of Mechanical Engineers, 1996.

12. A Heffron, JJR Williams, E Avital (2018), Numerical and experimental study of microvortex generators, AIAA J Aircraft, 55(6), 2256-2266.

13. Yashodhar, V., Humrutha, G., Kaushik, M., and Khan, S. (2017). Cfd studies on triangular micro-vortex generators in flow control. In IOP Conference Series: Materials Science and Engineering, volume 184, page 012007.

14. Paiboolsirichit, T. (2016). 3D simulation of wing fitted with Vortex Generators. 2016 Second Asian Conference on Defence Technology (ACDT).

15. Barrett, R., \& Farokhi, S. (1996). Subsonic aerodynamics and performance of a smart vortex generator system. Journal of Aircraft, 33(2), 393-398.

16. Chavez, M., Sanvido, S., Browne, O. M. F., \& Valero, E. (2017). Numerical and Parametric Study of MVGs on a UAV Geometry in Subsonic Flow. Computational Methods in Applied Sciences, 207-222.

17. ZHEN, T. K., ZUBAIR, M., \& AHMAD, K. A. (2011). Experimental and Numerical Investigation of the Effects of Passive Vortex Generators on Aludra UAV Performance. Chinese Journal of Aeronautics, 24(5), 577-583.

18. Lu, F. K., Li, Q., \& Liu, C. (2012). Microvortex generators in high-speed flow. Progress in Aerospace Sciences, 53, 30-45.

19. F. Menter. Zonal two equation kw turbulence models for aerodynamic flows. In 23rd fluid dynamics, plasmadynamics, and lasers conference, p. 2906, 1993.

20. Gao, L., Zhang, H., Liu, Y., \& Han, S. (2015). Effects of vortex generators on a blunt trailingedge airfoil for wind turbines. Renewable Energy, 76, 303-311.

21. Mueller-Vahl H, Pechlivanoglou G, Nayeri CN, Paschereit CO. Vortex generators for wind turbine blades: a combined wind tunnel and wind turbine parametric study. Am Soc Mech Eng 2012:899e914.

22. Ashill PR, Riddle GL, Stanley MJ. Control of threedimensional separation on highly swept wings. ICAS-94- 4.6.2, September 1994.

23. D. C. Wilcox, "Multiscale Model for Turbulent Flows," AIAA, vol. 26, no. 11, pp. 1311-1320, 1988.

24. F. R. Menter, "Improved Two-Equation k-omega Turbulence Models for Aerodynamic Flows," 1992.

25. ANALYSIS OF IMPLICIT LES METHODS

26. Grinstein, F. F., Margolin, L. G., \& Rider, W. J. (n.d.). A Rationale for Implicit LES. Implicit Large Eddy Simulation, 39-58.

27. C. Yao, J. Lin, and B. Allen. Flowfield measurement of device-induced embedded streamwise vortex on a flat plate. In 1st Flow Control Conference, p. 3162, 2002.

28. R. E. Sheldahl and P. C. Klimas. Aerodynamic characteristics of seven symmetrical airfoil sections through 180-degree angle of attack for use in aerodynamic analysis of vertical axis wind turbines. Technical report, Sandia National Labs., Albuquerque, NM (USA), 1981.

29. Fouatih, O. M., Medale, M., Imine, O., \& Imine, B. (2016). Design optimization of the aerodynamic passive flow control on NACA 4415 airfoil using vortex generators. European Journal of Mechanics - B/Fluids, 56, 82-96.

30. F. Balduzzi, A. Bianchini, R. Maleci, G. Ferrara, and L. Ferrari. Critical issues in the cfd simulation of darrieus wind turbines. Renewable Energy, 85:419-435, 2016. 